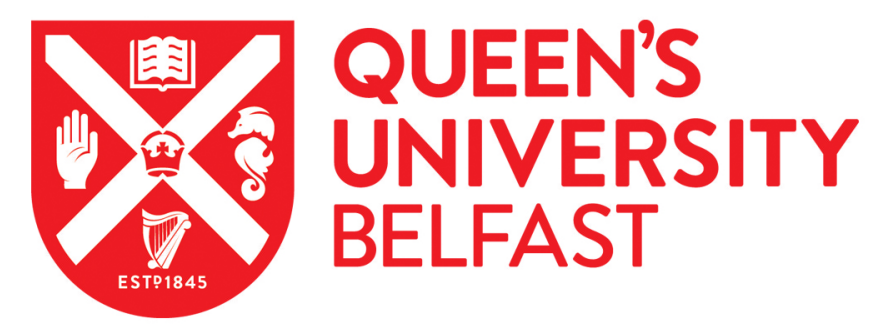

\title{
Modeling hydrodynamic cavitation in venturi: influence of venturi configuration on inception and extent of cavitation
}

Simpson, A., \& Ranade, V. V. (2018). Modeling hydrodynamic cavitation in venturi: influence of venturi configuration on inception and extent of cavitation. AIChE Journal. https://doi.org/10.1002/aic.16411

Published in:
AIChE Journal

Document Version:

Peer reviewed version

Queen's University Belfast - Research Portal:

Link to publication record in Queen's University Belfast Research Portal

Publisher rights

(c) 2018 American Institute of Chemical Engineers. This work is made available online in accordance with the publisher's policies. Please refer to any applicable terms of use of the publisher.

\section{General rights}

Copyright for the publications made accessible via the Queen's University Belfast Research Portal is retained by the author(s) and / or other copyright owners and it is a condition of accessing these publications that users recognise and abide by the legal requirements associated with these rights.

Take down policy

The Research Portal is Queen's institutional repository that provides access to Queen's research output. Every effort has been made to ensure that content in the Research Portal does not infringe any person's rights, or applicable UK laws. If you discover content in the Research Portal that you believe breaches copyright or violates any law, please contact openaccess@qub.ac.uk. 


\title{
Modelling Hydrodynamic Cavitation in Venturi: Influence of venturi configuration on inception \& extent of cavitation
}

\author{
Alister Simpson and Vivek V. Ranade* \\ School of Chemistry and Chemical Engineering \\ Queen's University Belfast, Belfast BT9 5AG, UK \\ *Email: V.Ranade@qub.ac.uk
}

\begin{abstract}
$\underline{\text { Abstract }}$
Hydrodynamic cavitation (HC) is useful for intensifying a wide variety of industrial applications including biofuel production, emulsion preparation and wastewater treatment. Venturi is one of the most widely used devices for hydrodynamic cavitation. Despite the wide spread use, the role and interactions among various design and operating parameters on generated cavitation is not yet adequately understood. This paper presents results of computational investigation into the cavitation characteristics of different venturi designs over range of operating conditions. Influence of the key geometric parameters like length of venturi throat and diffuser angle on the inception and extent of cavitation is discussed quantitatively. Formulation and numerical solution of multiphase computational fluid dynamics (CFD) models are presented. Appropriate turbulence model and cavitation model are selected and solved using commercial CFD code. Care was taken to eliminate influence of numerical parameters like mesh density, discretisation scheme and convergence criteria. The computational model was validated by comparing simulated results with three published data sets. The simulated results in terms of velocity and pressure gradients, vapour volume fractions and turbulence quantities etc. are critically analysed and discussed. Diffuser angle was found to have a significant influence on cavitation inception and evolution. The length of the venturi throat has relatively less impact on cavitation inception and evolution compared to the diffuser angle. The models and simulated flow field were used to simulate detailed timepressure histories for individual vapour cavities, including turbulent fluctuations. This in turn can be used to simulate cavity collapse and overall performance of hydrodynamic cavitation device as a reactor. The presented results offer useful guidance to the designer of hydrodynamic cavitation devices, identifying key operating and design parameters that can be manipulated to achieve the desired level of cavitational activity. The presented approach and results also offer a useful means to compare and to evaluate different designs of cavitation devices and operating parameters.
\end{abstract}

Key words: Hydrodynamic cavitation, venturi, CFD, multiphase, unsteady flows 
2 Hydrodynamic cavitation ( $\mathrm{HC}$ ) offers a potentially attractive route to process intensification across a wide range of industrial applications. For wastewater applications in particular, HC has been identified as a promising method for the removal of micropollutants (Ranade \& Bhandari [1]), and numerous recent studies have highlighted its potential to degrade a range of contaminants recalcitrant to conventional treatment methods [2-9]. Besides wastewater applications, the successful application of HC technology has been reported in a wide range of industrial processes, including biodiesel synthesis [10-12], bio-mass pre-treatment [13-15] and in food and beverage production [16-18] to name just a few. Reactor designs are typically of orifice or venturi type construction, and while the influence of reactor geometry has been reported to dictate overall performance to a significant extent (see for example $[19,20]$ ), the disparate range of geometries, target compounds and operating conditions in the open literature makes it generally difficult to draw firm conclusions on reactor $\&$ process design.

The cavitating venturi (CV) is a relatively simple flow device, widely used in passive flow control and metering applications. One characteristic is that above a specific pressure ratio a cavitating venturi becomes choked, beyond which the mass flow rate saturates at a certain level despite any increase in overall pressure ratio. As a result the CV found early application in rocket fuel systems [21], and the flow characteristics of CVs have been extensively studied experimentally and numerically [22-28]. Recent experimental studies have presented high speed visualization of cavitation inception and growth through individual venturi devices, such as that presented by Abdulaziz [29], Long et al. [30] and Brinkhorst et al. [31], each presenting a clear description of the evolution of cavitation and its effect on overall device operation in terms of flow rate and pressure drop. Cavitating venturi reactors have been the subject of a number of recent experimental studies, highlighting their promising effectiveness in a range of wastewater treatment [2,19,32] and other process intensification applications $[14,15]$. The role that the numerous interacting design parameters play in overall reactor performance however is still not fully understood and the reported experimental studies may employ nonoptimised operating conditions and venturi geometries. Sophisticated models have been developed for simulating collapse of a single cavity, generation of high pressure, temperature and hydroxyl radicals and subsequent reactions (for example, [2,9,33,34]). Capocelli et al. [35] have presented an integrated modelling approach to estimate reactor performance, which couples cavity dynamics systems to Bernoulli-type macroscopic flow calculations, along with estimations of turbulent fluctuations. This presents a useful framework on which to develop general, predictive models for cavitation reactors. The current state of the art models typically only consider the behaviour of single cavities however, and use simplified approximations to represent complex, macroscopic two phase flow behaviour through cavitation devices. As such, significant gaps exist in the knowledge base to aid the design and optimisation of a relatively simple cavitation device like the venturi. In this work we have attempted to fill some of these gaps.

In this study we develop and use multiphase Computational Fluid Dynamics (CFD) models to simulate flow through a systematic range of venturi designs, in order to develop insights into the role that key operating and 
geometric parameters have on the resulting two-phase flow field. Particular attention is given to cavitation

2 inception and evolution. In developing the modelling approach, comparisons have been made with three experimental configurations, summarized in Table 1, so as to test the ability of the computational approach to replicate overall flow characteristics for cases featuring a range of different geometries and operating conditions. Case 1 is based on the experimental data presented by Brinkhorst et al. [31], which provides data for a relatively large $11 \mathrm{~mm}$ throat venturi in the choked cavitation regime; Case 2 is based on data presented by Abdulaziz, [29], which considers a small $3.6 \mathrm{~mm}$ diameter throat venturi operating into the choked cavitation regime, and Case 3 is based on experimental work by Musmarra et al. [2,3], which operates below choked conditions. Using the geometry and operating conditions for Case 3 as a baseline, the computational approach is then extended to investigate the cavitation characteristics of a series of venturi designs having varying throat length to diameter ratio and diffuser angle.

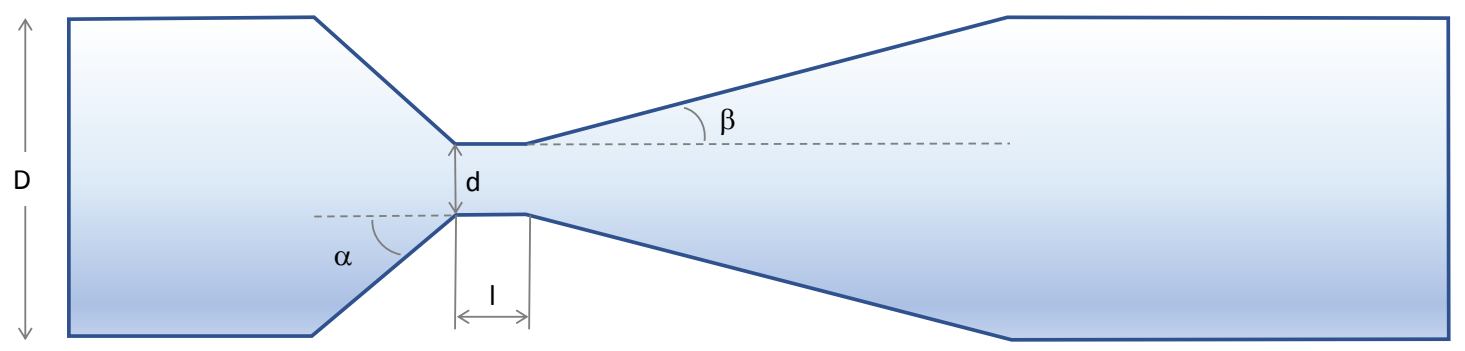

Figure 1: Schematic of venturi

Table 1: Venturi configurations

\begin{tabular}{|l|c|c|c|c|c|c|c|c|c|}
\cline { 2 - 10 } \multicolumn{1}{c|}{} & Case 1 [29] & Case 2 [31] & Case 3 & $\mathrm{l} / \mathrm{d}=1$ & $\mathrm{l} / \mathrm{d}=\mathbf{2}$ & $\mathrm{l} / \mathbf{d}=\mathbf{3}$ & $\boldsymbol{\beta}=\mathbf{7 . 5 ^ { \circ }}$ & $\boldsymbol{\beta}=12.5^{\circ}$ & $\boldsymbol{\beta}=15^{\circ}$ \\
\hline$D[\mathrm{~mm}]$ & 20 & 25.5 & 12 & 12 & 12 & 12 & 12 & 12 & 12 \\
\hline$d[\mathrm{~mm}]$ & 3.6 & 11.2 & 2 & 2 & 2 & 2 & 2 & 2 & 2 \\
\hline$I[\mathrm{~mm}]$ & 9 & 11.2 & 0 & 2 & 4 & 6 & 0 & 0 & 0 \\
\hline$\alpha\left[^{\circ}\right]$ & 6.2 & 22.5 & 22.5 & 22.5 & 22.5 & 22.5 & 22.5 & 22.5 & 22.5 \\
\hline$\beta\left[^{\circ}\right]$ & 6.2 & 3.5 & 9.25 & 9.25 & 9.25 & 9.25 & 7.5 & 12.5 & 15 \\
\hline
\end{tabular}

\section{Mathematical models}

In venturi or similar linear flow restriction, cavitation occurs when the flow rate attained is sufficient to drive local pressures within the throat of the device down to the saturated vapour pressure of the liquid. Cavitation inception is marked by an initial transition from a single-phase flow to a two phase bubbly flow, and as flow rate increases an increasingly complex flow field develops. Flow fields are typically highly turbulent; larger gas filled vapour structures form, grow, and trigger vortex breakup. Discrete cavities can undergo oscillatory growth, coalescence and break up before finally collapsing as they are transported into higher pressure regions. The spatial and temporal timescales over which these events occur span a wide range, and as such modelling cavitation is a particularly complex task. The most fundamental approach is to apply Direct Numerical Simulation 
1 (DNS), which resolves the smallest scales of turbulence and cavity evolution. However the extreme

2 computational demands limit this approach to the study of relatively small fluid volumes and bubble quantities

3 [36]. Considering that the focus of this work is on carrying out large number of simulations for a wide range of

4 design and operating parameters, we used RANS (Reynolds Averaged Navier-Stokes) approach with appropriate

5 turbulence model. A pseudo-homogeneous, or a mixture model, is used in which the working medium is treated

6 as a single fluid composed of a homogenous mixture of two phases, with appropriate relationships defined to

7 drive mass transfer. The following sections (2.1 and 2.2) describe the model equations representing the

8 cavitating two-phase flow field with phase change. Besides obtaining the time averaged flow field, it is useful to simulate transient trajectories of cavities within the flow domain to gain insight about the time-pressure histories experienced by cavities as they are transported through the device. To achieve this, the Eulerian mixture computations were coupled to the Lagrangian simulations for discrete cavity trajectories. The model equations for these Lagrangian simulations are discussed in Section 2.3.

\subsection{Flow \& turbulence models}

The working medium is treated as a single fluid, comprised of a homogeneous mixture of two phases. The continuity equation for the mixture flow is written as:

$$
\frac{\partial}{\partial t}\left(\rho_{m}\right)+\nabla \cdot\left(\rho_{m} \vec{v}_{m}\right)=0
$$

Where $\rho_{m}$ is the mixture density, $\& \vec{v}_{m}$ is the mass-averaged velocity. The corresponding momentum equation for the mixture flow, ignoring drift velocities, is written as:

$$
\frac{\partial}{\partial t}\left(\rho_{m} \vec{v}_{m}\right)+\nabla \cdot\left(\rho_{m} \vec{v}_{m} \vec{v}_{m}\right)=-\nabla p+\nabla \cdot\left[\mu_{m}\left(\nabla \vec{v}_{m}+\nabla \vec{v}_{m}^{T}\right)\right]+\rho_{m} \vec{g}+\vec{F}
$$

In Reynolds averaged (RANS) approaches, the velocity terms in Equations (1) \& (2) are replaced by the sum of their mean and instantaneous components, $u=\bar{u}+u^{\prime}$, and an ensemble average is taken. This averaging process results in additional terms representing the effects of turbulence. These additional terms take the general form $\partial / \partial x_{j}\left(-\rho \overline{u_{\imath} u_{\jmath}}\right)$, and are known as the Reynolds stresses. In order to close the momentum equation, the introduced Reynolds stress terms require additional mathematical models. One approach is to use a Reynolds Stress Model (RSM), which involves solving separate transport equations for each of the additional Reynolds stresses (6 in total for 3D cases). More typical in RANS approaches is to employ the Boussinesq hypothesis, which relates the Reynolds stresses to the mean velocity gradients in the flow as:

$$
-\rho \overline{u_{\imath} u_{\jmath}}=\mu_{t}\left(\frac{\partial u_{i}}{\partial j}+\frac{\partial u_{j}}{\partial i}\right)-\frac{2}{3}\left(\rho k+\mu_{t} \frac{\partial u_{k}}{\partial k}\right) \delta_{i j}
$$




$$
\mu_{t}=\alpha^{*} \frac{\rho k}{\omega}
$$

$$
\frac{\partial}{\partial t}(\rho \omega)+\frac{\partial}{\partial x_{i}}\left(\rho \omega u_{i}\right)=\frac{\partial}{\partial x_{j}}\left(\Gamma_{\omega} \frac{\partial \omega}{\partial x_{j}}\right)+G_{\omega}-Y_{\omega}+S_{\omega}
$$

$G$ represents the generation, $\Gamma$ the effective diffusivity, and $Y$ is the dissipation due to turbulence [38]. $S_{k}$ $\& S_{w}$ are user-defined source terms.

\subsection{Cavitation model}

Various mass transfer models have been proposed to describe the cavitation process, with the most commonly used approaches based on reduced forms of the Rayleigh-Plesset equation. Examples include the models proposed by Schnerr \& Sauer [39], Zwart et al. [40] and Singhal et al. [41]. The computational work described in this paper is based on the latter cavitation model developed by Singhal; this has been validated against a wide range of flow cases (see examples in [41], [42]), and offers the advantage that the bubble number per unit volume, $n$, need not be prescribed as input. In this model, the vapour volume fraction is computed locally from a transport equation for the vapour mass fraction, $f$, which introduces an additional pair of mass source and sink terms for the evaporation $\left(R_{e}\right)$ and condensation $\left(R_{c}\right)$ of the vapour:

$$
\frac{\partial}{\partial t}\left(\rho_{m} f\right)+\nabla \cdot\left(\rho_{m} \vec{v}_{m} f\right)=\nabla \cdot(\Gamma \nabla f)+R_{e}-R_{c}
$$


1

Where:

$$
\frac{1}{\rho_{m}}=\frac{f}{\rho_{v}}+\frac{1-f}{\rho_{l}}
$$

2

3 can then be calculated as follows:

$$
\alpha=f \frac{\rho_{m}}{\rho_{v}}
$$

6

$$
\frac{\partial}{\partial t}\left(\rho_{m} f\right)+\nabla \cdot\left(\rho_{m} \vec{v}_{m} f\right)=(n 4 \pi)^{1 / 3}+(3 \alpha)^{1 / 3} \frac{\rho_{v} \rho_{l}}{\rho}\left[\frac{2}{3}\left(\frac{P_{B}-P}{\rho_{l}}\right)\right]^{1 / 2}
$$

9

Equation (10) is referred to as the Reduced Bubble Dynamics Formulation. In this expression, all terms except $\mathrm{n}$, the bubble number density, are either known constants or dependent variables. To avoid having to specify a bubble number density, the phase change expression is rewritten in terms of bubble radius:

$$
R_{e}=\frac{3 \alpha}{R_{B}} \frac{\rho_{v} \rho_{l}}{\rho_{m}}\left[\frac{2}{3}\left(\frac{P_{B}-P}{\rho_{l}}\right)\right]^{1 / 2}
$$

The typical bubble size, $R_{B}$, is taken to be equal to the limiting (maximum possible) bubble size using a correlation commonly used in the nuclear industry:

$$
R_{B}=\frac{0.061 W e \sigma}{2 \rho_{l} v_{r e l}^{2}}
$$

Where We is the Weber number, and $\sigma$ is the surface tension. In the Singhal model, the square of the relative velocity term is approximated as $v_{r e l}^{2}=v_{c h}=\sqrt{k}$ (In bubbly flows the phase change rate is proportional to $v_{r e l}^{2}$, however most practical two-phase flow regimes display a linear dependence). The relative velocities and turbulent velocity fluctuations are of the same order (1-10\%), and as such $\sqrt{k}$ is considered to be a suitable approximation for $v_{c h}$. This produces the following final pair of phase change rate terms:

$$
R_{e}=C_{1} \frac{\max (1.0, \sqrt{k})}{\sigma} \rho_{v} \rho_{l}\left[\frac{2}{3}\left(\frac{P-P_{v}}{\rho_{l}}\right)\right]^{1 / 2}\left(1-f_{v}-f_{g}\right)
$$




$$
R_{c}=C_{2} \frac{\max (1.0, \sqrt{k})}{\sigma} \rho_{v} \rho_{l}\left[\frac{2}{3}\left(\frac{P-P_{v}}{\rho_{l}}\right)\right]^{1 / 2} f_{v}
$$

1

2

$$
\begin{aligned}
& P_{\text {turb }}^{\prime}=0.39 \rho_{m} k \\
& P_{v}=P_{\text {sat }}+P_{\text {turb }}^{\prime} / 2
\end{aligned}
$$

Where $\rho_{\mathrm{m}}$ is the mixture density, $k$ is the turbulent kinetic energy, and the constant value of 0.39 is taken from [41].

\subsection{Discrete phase model (DPM)}

Trajectories of individual cavities were simulated using the Lagrangian approach. Since the two-phase flow field is already computed using the models described above, one-way coupling was assumed between the discrete cavities and the continuous mixture while simulating cavity trajectories. The cavity trajectories are driven by the primary flow gradients and turbulence quantities. The particle trajectories are computed by integrating the force balance for a discrete particle of a series of discrete time steps; the force balance is given as:

$$
\frac{d \vec{u}_{p}}{d x}=\frac{\vec{u}-\vec{u}_{p}}{\tau_{r}}+\frac{\vec{g}\left(\rho_{p}-\rho\right)}{\rho_{p}}+\vec{F}
$$

This equates the particle inertia with the forces acting on the particle. The first term on the right-hand side of the equation is the drag force per unit mass of the particle, and the second term is the force due to gravity. The final term, $\vec{F}$, is an additional acceleration term. In the present study, the particles are treated as massless flowfollowers. The influence of continuous phase turbulence on the tracked particles is accounted for by separating the velocity, $u$, into mean and instantaneous components:

$$
u=\bar{u}+u^{\prime}
$$

In the work presented here, the discrete random walk model, or "eddy lifetime" model is used to include the effects of turbulence on the discrete cavity trajectories [38]. In this approach, each discrete particle is considered 

involves introducing two modelled terms; firstly, the random fluctuating component of velocity is calculated as a function of the local turbulent kinetic energy value:

$$
u^{\prime}=\zeta \sqrt{2 k / 3}
$$

Where $\zeta$ is a normally distributed random number. Secondly, the concept of a particle eddy lifetime, $T_{L}$, is introduced to define the time intervals over which this random fluctuating component is updated. This "eddy lifetime" is approximated as a function of the local turbulence frequency:

$$
T_{L} \approx 0.15 \frac{k}{\varepsilon}
$$

Additional limits can be placed on the maximum time step size; in this study a minimum of 5 time steps is also imposed across any given computational cell. Using time-averaged velocities, pressures and turbulence quantities from the solved Eulerian flow field, discrete cavities were the initialised on an iso-surface of volume fraction equal to 1 ; the edge of the predicted vapour filled cavity. The minimum total sampling time was set to be equal to the time required for the pressure to recover to the back pressure. Initially a suitably large number of particle trajectories were computed (of the order of 100), and from these a sample of 10 trajectories were selected and averaged for post-processing and visualization.

\section{Results \& Discussion}

\subsection{Numerics and convergence strategy}

The model equations described in the preceding section were all solved using commercial CFD code, Ansys Fluent (v17). 2D axi-symmetric models were used throughout, and in each case the pressure ratio was fixed by inlet and outlet pressure boundary conditions, making the flow-rate solution dependent. Initially single-phase calculations were carried out, and the cavitation model was then subsequently activated using the solved single-phase results as initial conditions. The SIMPLE algorithm was used for pressure velocity coupling [43], with the PRESTO! discretisation scheme applied for pressure. For the momentum, pressure and turbulent quantities $2^{\text {nd }}$ order discretization was applied in each instance.

The $k$ - $\omega S S T$ turbulence closure model was selected for use throughout. This model has been shown to offer improved accuracy over other eddy-viscosity based models in a number of comprehensive validation studies of complex flow cases involving separation and adverse pressure gradients [44]. The interaction between cavitation and turbulent flow structures is extremely complex, and the detailed mechanisms are not yet fully understood. The difficulties in turbulence modelling in cavitating flows and proposed approaches have been described elsewhere in [45-47]. Reboud et al. [45] made the argument that the standard k- $\varepsilon$ model tends to produce high turbulent viscosity $\left(\mu_{t}\right)$ predictions in the cavity wake, resulting in an underprediction of the reentrant jet, and thus resulting in a stable cavity. To generate the prediction of unsteady cavity shedding, the authors applied an arbitrary pre-multiplier to limit the turbulent viscosity. Examples exist in open literature of 
favourable correlations being obtained between models applying this Reboud limiter with experimental data, and also with eddy resolving turbulence models such as the scale adapted simulation (SAS) model [47]. The arbitrary nature of the correction factor however means that it is likely to be dependent on geometry, and in lieu of sufficient experimental data to "tune" the turbulence model no such limiter has been applied in the present study.

Although the results presented in the present work do not exhibit resolved vapour shedding, in the cavitating regime the simulations highlighted the formation of a fluctuating pressure field. This results from the unsteady evolution of the vapour cavity downstream of the throat, and as such a transient URANS approach was used throughout the present work to model the cavitating flow regimes. Beyond cavitation inception, the predicted vapour pocket shows a quasi-periodic growth and collapse, with an initially high oscillation frequency which was predicted to decrease gradually with increasing pressure ratio. Figure $2 a$ presents sampled velocity fluctuations half-way along the diffuser section, showing the change in both frequency and amplitude of oscillation with varying device pressure ratio. The corresponding growth and collapse of the vapour pocket is shown in Figure $2 b$ for a device pressure ratio of 4 . Oscillation frequencies were found to lie in the $100-700 \mathrm{hz}$ range, which is general agreement with measured observations in open literature $[25,28]$, as well as results from numerical studies [23]. Time step sizes necessary to obtain convergence for the URANS calculations therefore varied from $1 \times 10^{-4}$ to $1 \times 10^{-6} \mathrm{~s}$.

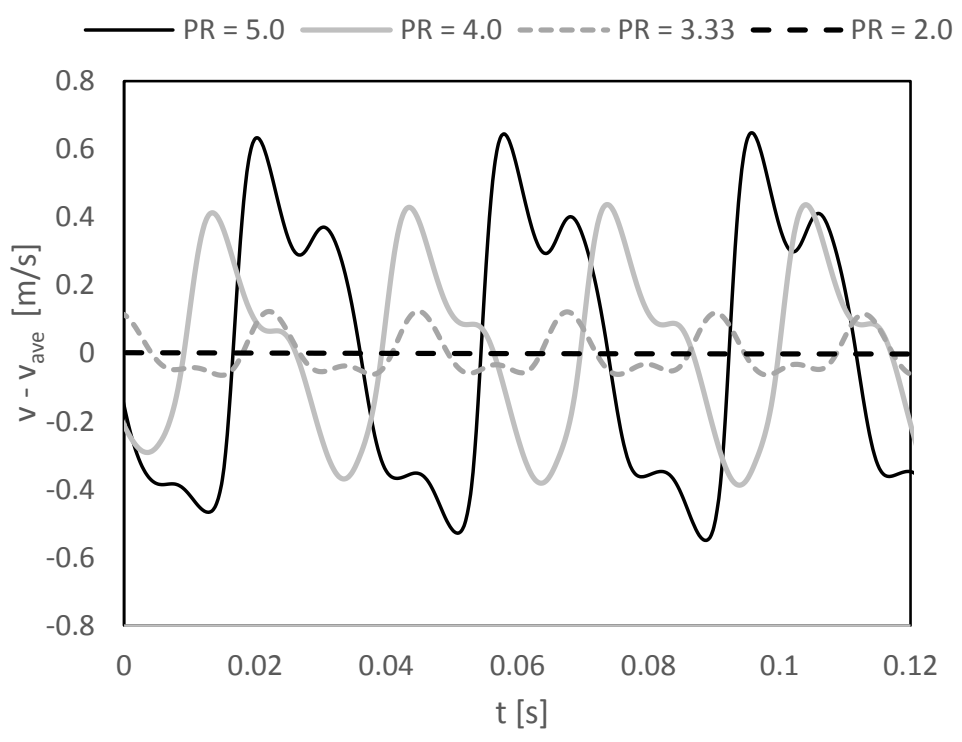

(a) Velocity fluctuations in diffuser mid-plane

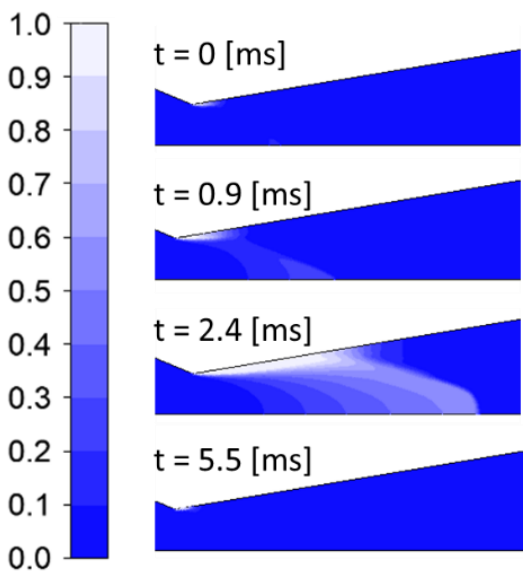

(b) Vapour volume fraction $(\operatorname{Pr}=4.0)$ Figure 2: Fluctuations in velocity and vapour volume fraction in diffuser section

To determine the sensitivity to grid refinement, particularly local cell sizes and growth ratios in the orifice throat, a series of $4 x$ systematically refined meshes were investigated. Grid sizes of 20,000 cells; 40,000; 80,000 and a final grid of 160,000 cells were constructed, all using 2D quadrilateral elements, and converged results obtained from each. Figure $3 b$ : a shows a sample grid with a close up view of the working section, and Figure $3 b$ : a presents plots of turbulent kinetic energy and axial velocity distributions half way along the diffuser section with 
varying grid densities. There is a negligible difference in profiles between grid sizes of 80,000 and 160,000 , and as such the grid refinement was set at 80,000 cells. This cell count featured a first near wall cell height of $2 \mathrm{e}-$ $3 \mathrm{~mm}$, with a maximum element size of $0.05 \mathrm{~mm}$ specified in the throat of the venturi. The variation in predicted wall $\mathrm{y}^{+}$for a selection of the grids studied are presented in the supplementary information in Figure SI1, along with a sample mesh illustration in Figure SI2. For the subsequent parametric study, the same mesh settings were translated onto the different geometries such that the same refinement levels were maintained in the $x$ and $y$ - directions, which resulted in increased mesh counts with increasing throat length.

8

Figure 3a: Sample mesh \& applied boundary conditions

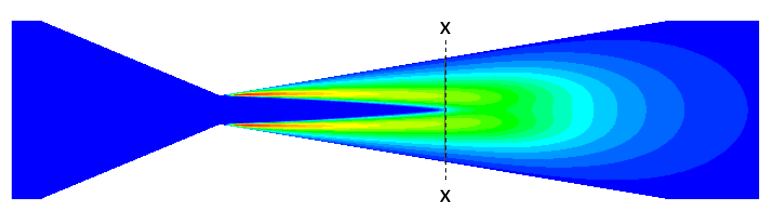

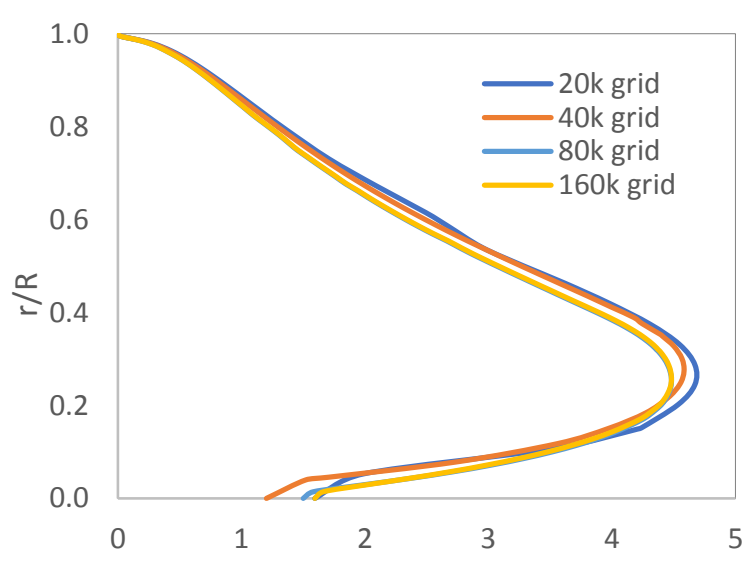

Turbulent kinetic energy, $\mathrm{k}\left[\mathrm{m}^{2} / \mathrm{s}^{2}\right]$

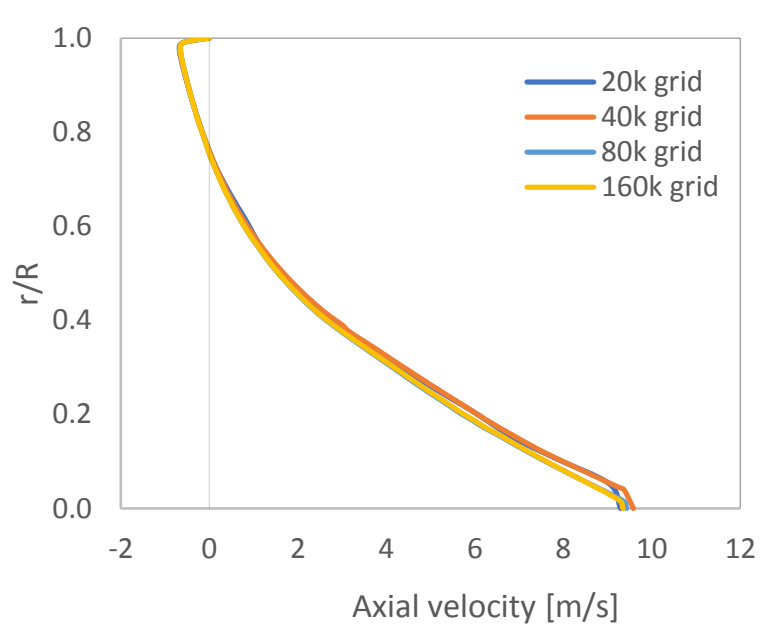

Figure 3b: Predicted turbulence and velocity profiles with varying mesh density (Case 3 )

Figure 3: Computational grid \& sensitivity study

\subsection{Comparison with experimental data}

Figure 4 presents a comparison of predicted and measured flow rate versus pressure drop for Case 1, showing predictions with and without the cavitation model activated. This confirms the capability of the cavitation model to replicate the overall flow characteristics of the device in the cavitating regime, with cavitation shown 
to produce a reduction in flow rate. Error bars have been omitted for clarity, however the absolute predictions

2 in flow rate all sit within $2 \%$ of the measured data. Experimental comparisons are provided for Case 2 in Figure

$35 a$, again showing good agreement with both the overall mass flow - pressure drop relationship, in this case

4 within $5 \%$ of measured data. The point at which choked cavitation occurs is again well replicated by the model

5 at different pressure levels. Figure $5 b$ shows the predicted and measured minimum throat pressures, showing

6 good agreement accounting for variations in non-condensable gas content, which is neglected in the

7 computational approach. This provides added confidence in the ability of the model to reliably capture the

8 cavitation inception point. The final experimental comparison for Case 3 is presented in Figure 6, which shows

9 close correlation across the cavitating range (again within $2 \%$ of measured flow rate data), which in this instance

10 is below the choking limit.

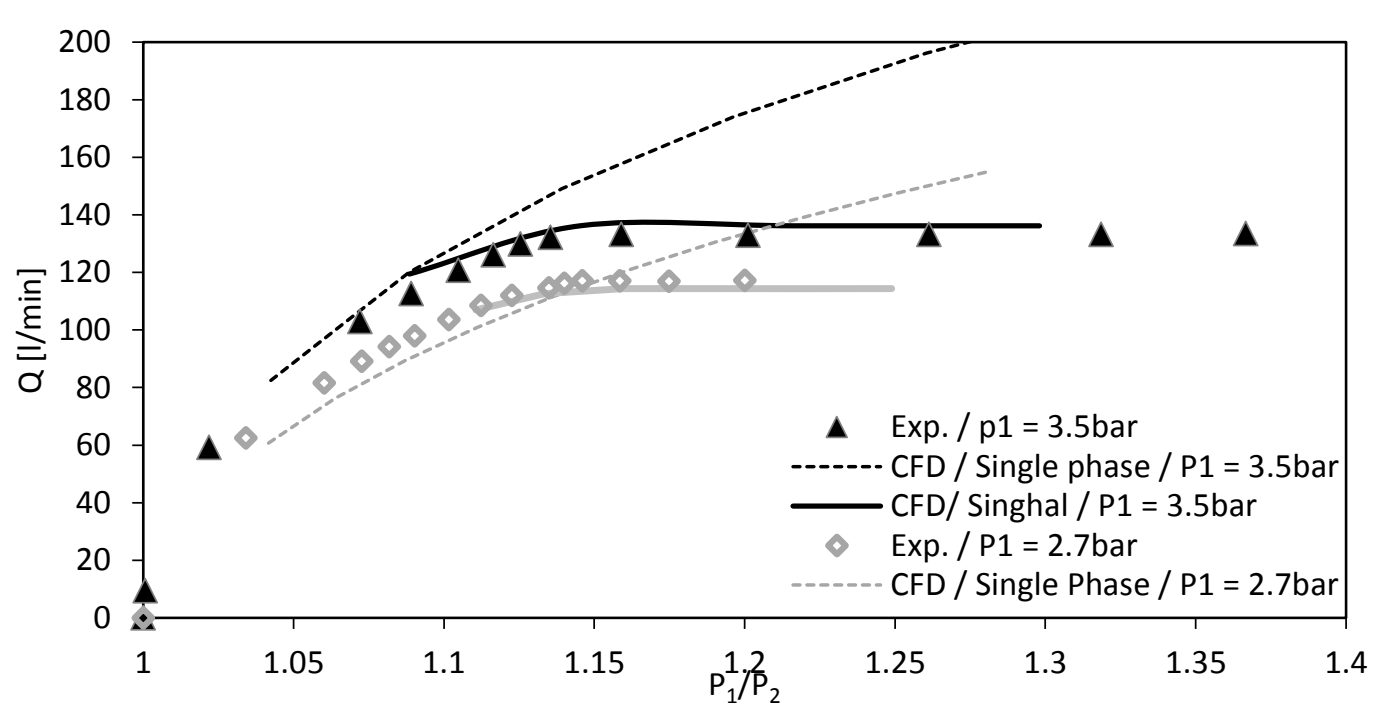

Figure 4: Case 1; Measured \& predicted flow rate vs pressure ratio, Case 1

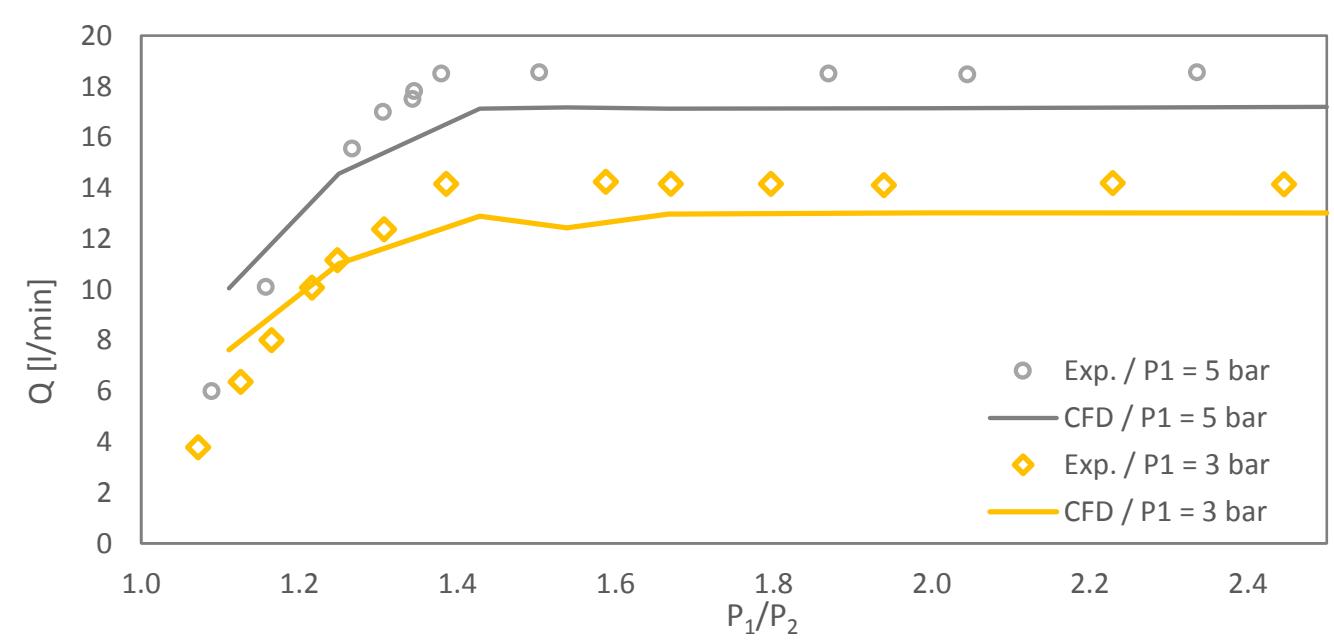

Figure 5a: Case 2; Measured \& predicted flow rate vs pressure ratio 


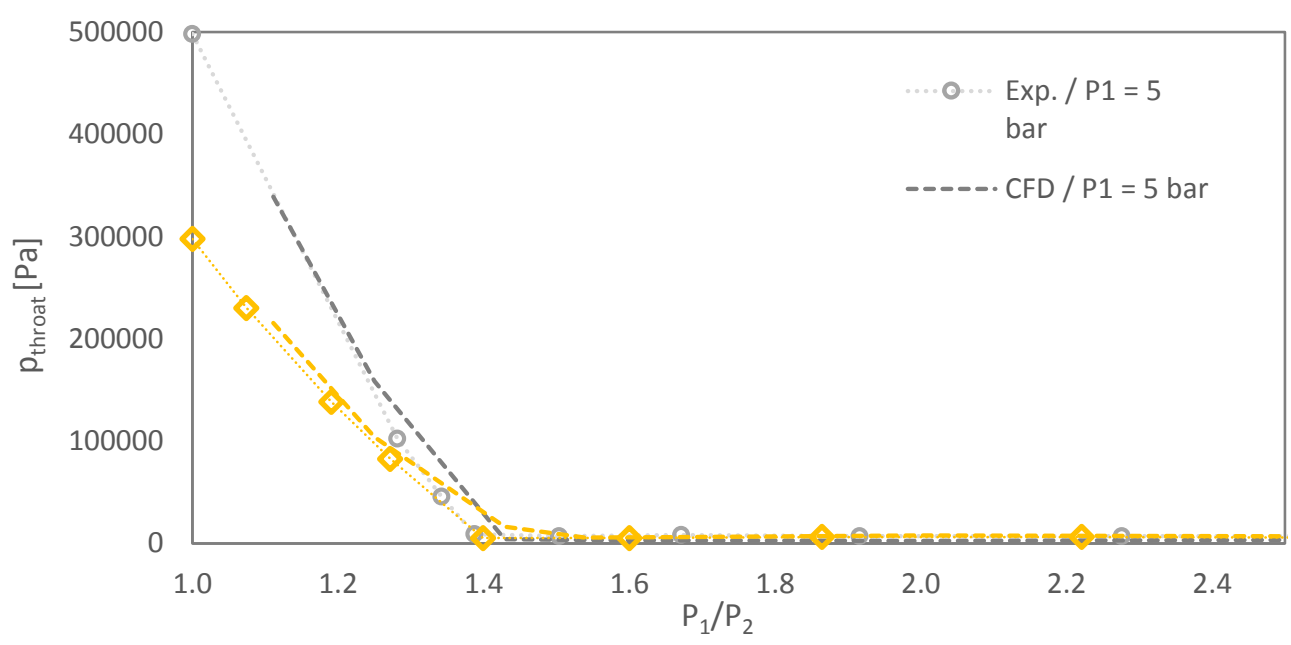

Figure 5b: Case 2; Measured \& predicted minimum throat pressure vs pressure ratio

Figure 5: Experimental comparisons, venturi case 2

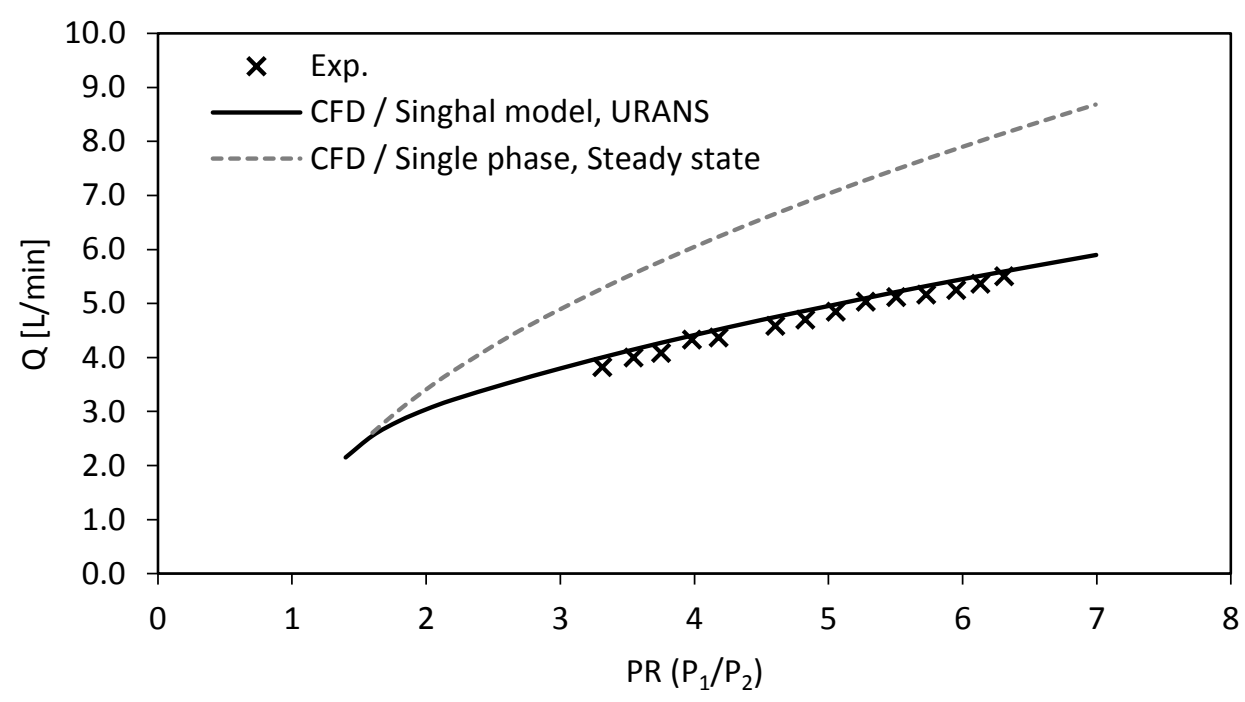

Figure 6: Case 3; Measured \& predicted flow rate vs pressure ratio

\subsection{Influence of device pressure ratio}

Figure 7 shows the predicted static pressure distribution both along the outer wall and the axis for Case 3 . Cavitation inception is predicted to occur at a pressure ratio of 1.6 , or a cavitation inception number $\sigma_{i}$ of 0.96 , which is in line with experiment. Inception is initially localised around the outer wall of the venturi throat as a separation bubble is formed, and the low-pressure region then extends axially and also radially inwards with increasing pressure ratio. As shown in Figure $7 \mathrm{~b}$, as device pressure ratio approaches 4.0 the vapour cavity reaches the axis of the venturi, and a prominent pressure spike is observed in the plotted results; this sharp pressure rise corresponds to edge of the vapour cavity. The time-averaged 2D pressure distributions at pressure ratios of 1.6 and 4.0 are shown in detail in Figure $8 a$ and Figure $8 b$ respectively, with the evolution of the vapour cavity shown in Figure $8 c$. 


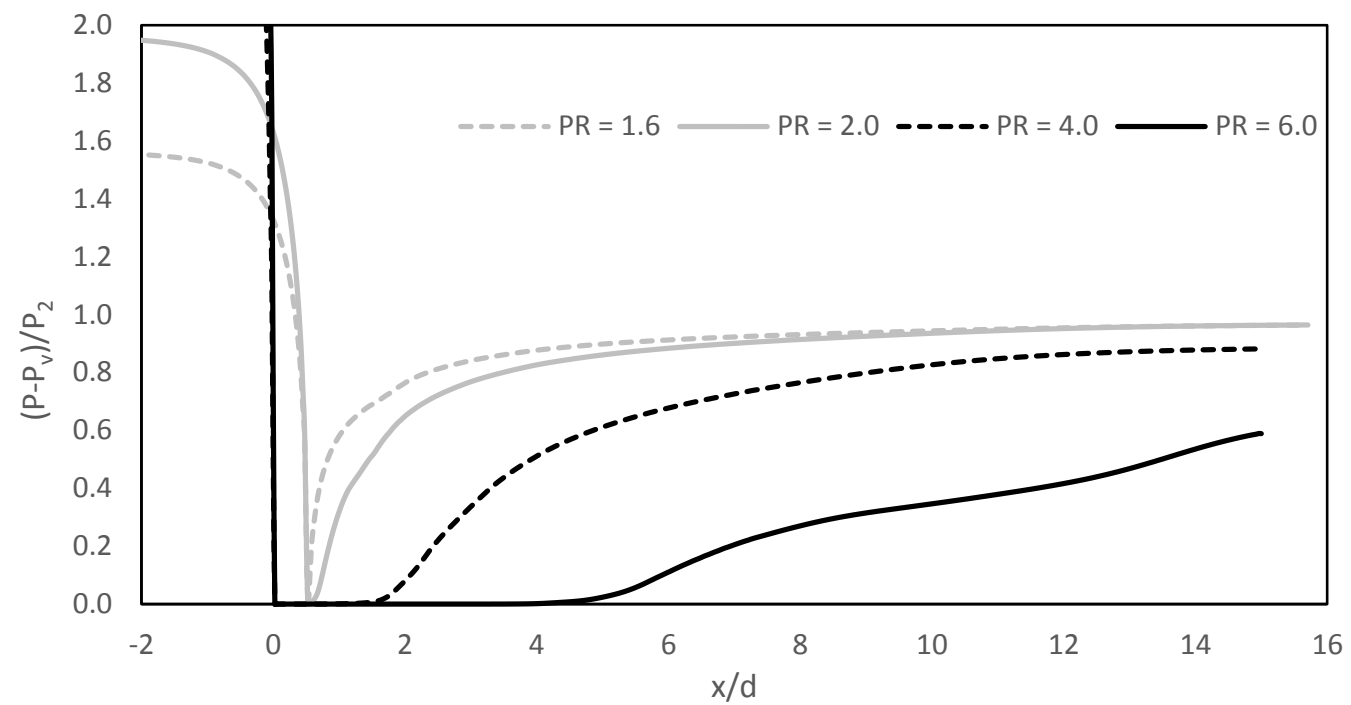

3

Figure 7a: Predicted pressure vs distance along outer wall,

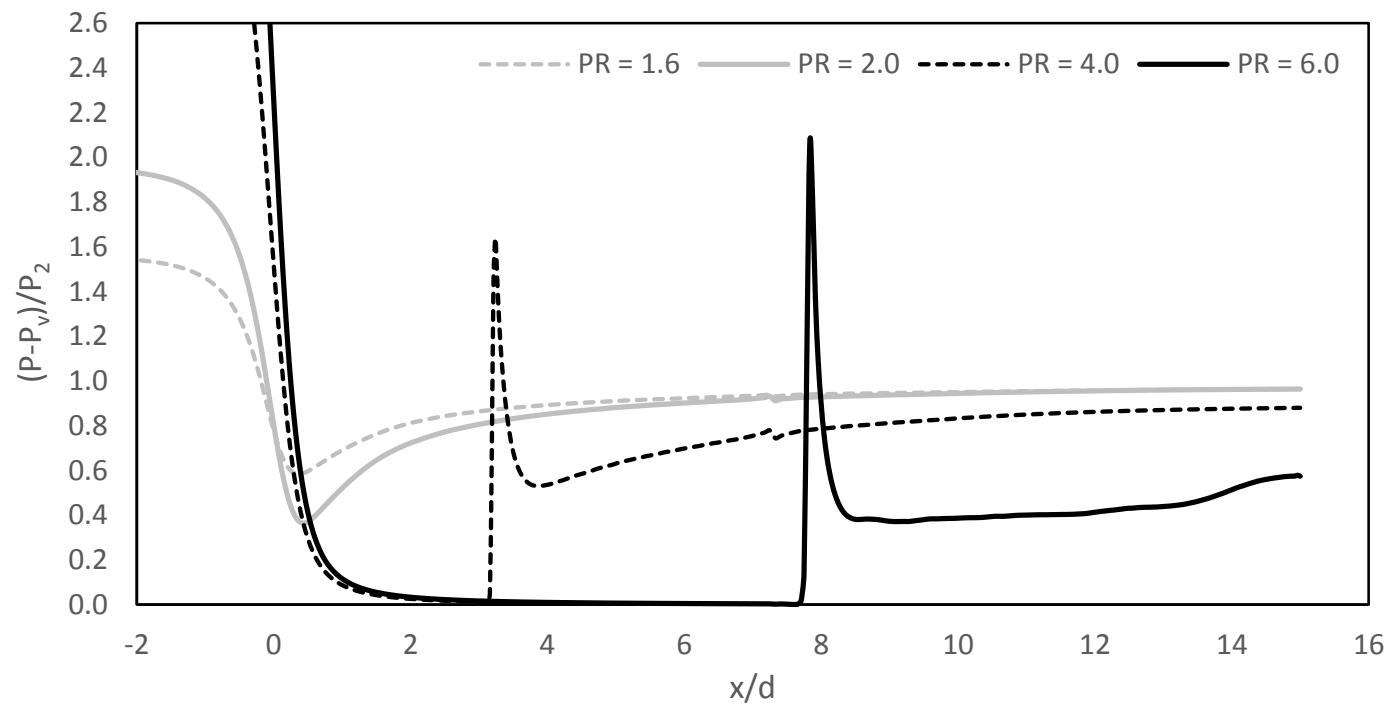

Figure 7b: Predicted pressure vs distance along axis

Figure 7: Case 3, predicted pressure vs distance profiles 


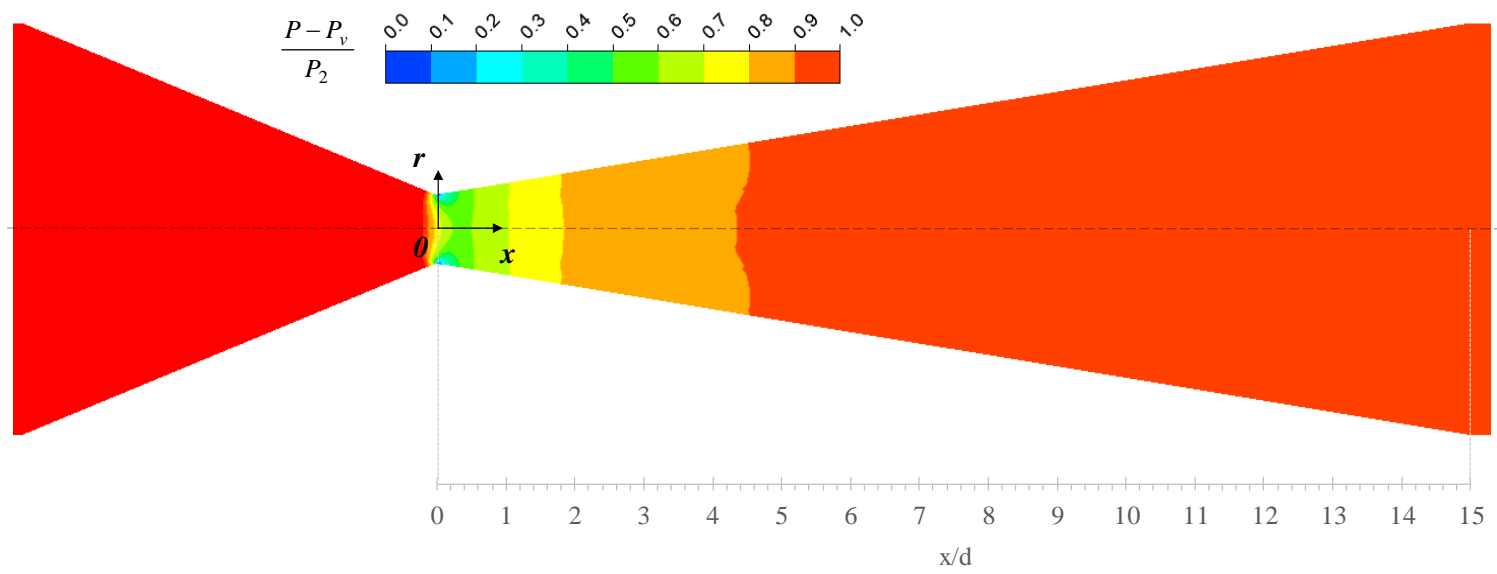

Figure 8a: Non-dimensional static pressure @ PR=1.6 (Inception point)

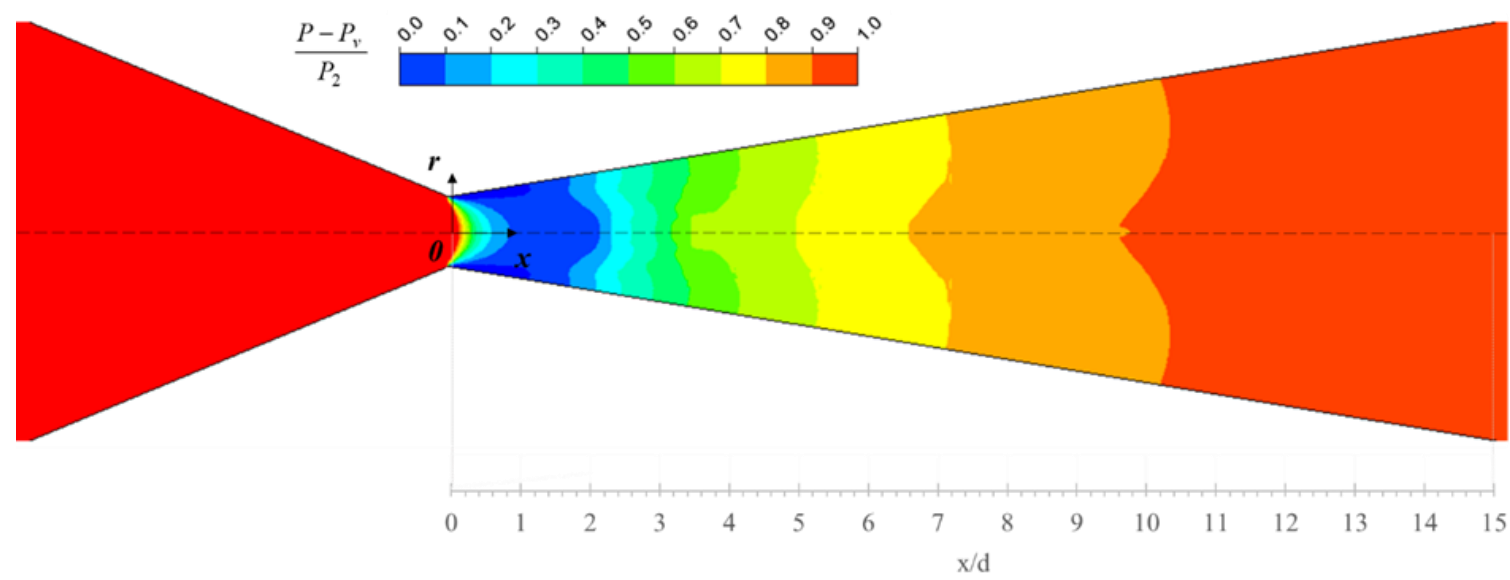

Figure 8b: Non-dimensional static pressure @PR =4.0

Figure 9Error! Reference source not found. presents the variation in simulated oscillation frequency and Strouhal number with Reynolds number. For each simulation, the predicted unsteady velocities at diffuser exit were sampled, and the peak frequencies extracted from power spectrum (via Fast Fourier Transforms [FFT]) (See 
is increased, with a corresponding increase in oscillation amplitude. This is qualitatively in line with previous experimental \& numerical investigations in open literature; Sayyaadi [25] for example has reported unsteady measurements in a cylindrical cavitating venturi, in which the formation of a quasi-periodic vapour cavity, which grows and retracts at frequencies of the order of $25-250 \mathrm{hz}$ is described. The oscillation frequencies were observed to increase with increasing flow rate / decreasing cavitation number. The corresponding amplitudes were found to show the opposite trend, increasing with cavitation number. Similar measurements have been presented by Sato et al. [28], who found frequencies in the 400hz range. Xu et al. in 2002 [23] reported a numerical investigation of cavitating venturi flows in both cryogenic fluids and in water, presenting results which replicated the formation of a quasi-periodic cavity which expended and retracted downstream of the throat. Oscillation frequencies of the order of $400 \mathrm{hz}$ were predicted, with peak frequencies also observed to increase with decreasing cavitation number. It is interesting to note that there appears to be two distinct regimes in variation of frequency with Reynolds number. The slope of variation of frequency versus Re reduces at PR of about 3 , where the pressure in the venturi approaches the vapour pressure across the full cross section. At this point there is a large density difference between the throat and diffuser outlet (from $\rho_{v} \sim 0.5 \mathrm{~kg} / \mathrm{m} 3$ at the throat to $\rho_{\mathrm{I}} \sim 1000 \mathrm{~kg} / \mathrm{m} 3$ at diffuser exit), as well as a large gradient in viscosity. At this point a complex multiphase flow field develops, with significant gradients present in both density and viscosity between the throat and diffuser outlet. The underlying reasons for the change in frequency are likely to be a similarly complex function of flow structures; a recent experimental paper by Long and co-workers [30] shows a similar transition in flow structure at the point where the vapour cavity merges at the axis of the venturi, after which the time-average length of the vapour cavity was found to proceed more rapidly. The frequencies of this oscillation mechanism are of the order of $100 \mathrm{~Hz}$, whereas the turbulent frequencies are of the order of $10 \mathrm{kHz}$, so turbulence effects can be considered to dominate the intensity of cavitation in terms of final collapse conditions.

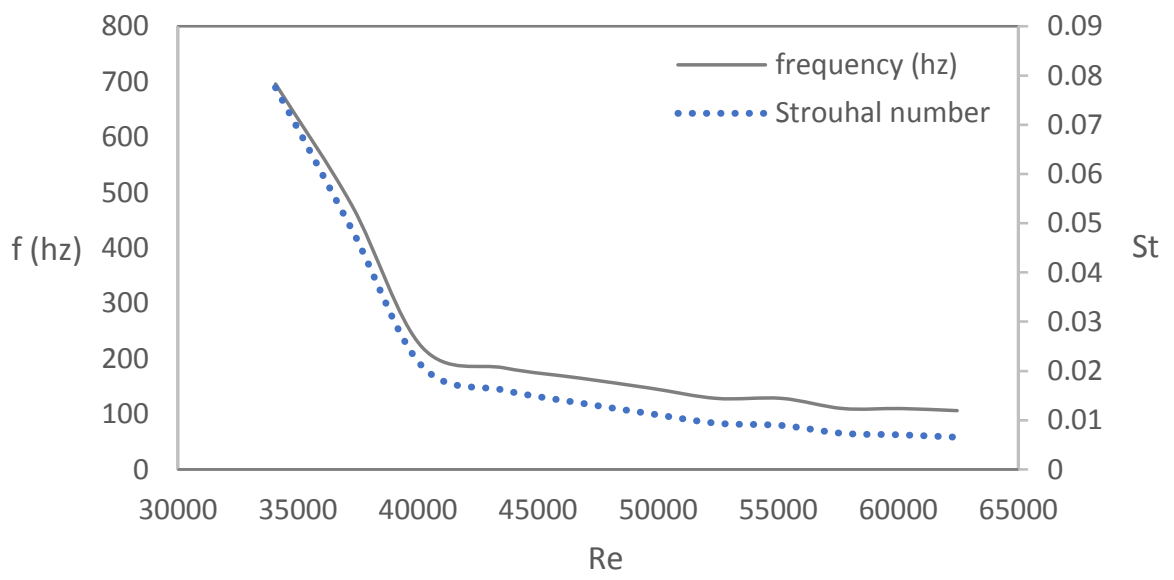

Figure 9: Case 3, predicted oscillation frequencies \& amplitudes vs pressure ratio

\subsection{Discrete cavity results - Case 3}

Using the converged solutions of the Eulerian multiphase flow fields, cavity trajectories were simulated to gain insight into the turbulent pressure fields experienced by the cavities generated at the restriction. In each case, 
the trajectory calculations were initialized from a surface of constant vapour volume fraction $=1.0$, representing the edge of the predicted gas filled cavity in the venturi. For each trajectory, the mean pressures and turbulence quantities were processed, and the fluctuating component of pressure, $p^{\prime}$, calculated as a function of a normally distributed random number as follows:

$$
p^{\prime}=\bar{p}+\zeta \rho k
$$

5

6

Discrete cavity trajectories for the Case 3 are shown in Figure 10a along with the computed turbulent kinetic energy for a pressure ratio of 2.0. The cavities initiate at the edge of the vapour pocket in the venturi throat, and then interact with the turbulence generated at the edge of the filled vapour pocket and the shear layer formed at the edge of the liquid jet. Turbulence frequencies are orders of magnitude higher than the predicted oscillations in the Eulerian flow field results, and as such it was deemed suitable to restrict calculations to steady particle tracks. The resulting time - pressure histories of averaged cavity trajectory sets are plotted in Figure $10 b$, along with the turbulent fluctuating pressure component, $p^{\prime}$, highlighting the variation with driving pressure ratio. The raw time-history data prior to averaging for pressure ratios of $3.0 \& 4.0$ is presented in Figures SI.3 \& SI.4. As pressure ratio is increased, the cavities experience an increasingly delayed pressure recovery, characterised by a longer low pressure region with lower turbulent frequencies. The turbulent frequencies dominate over the predicted pressure oscillations in the solved continuous flow field by at least an order of magnitude throughout the lifetime of the trajectories for each case, and as such the turbulent frequencies will have the dominant effect on individual cavity dynamics. At a pressure ratio of 3.0, the cavities are subjected to relatively high frequency fluctuations over their initial lifetime, whereas at pressure ratios of 5.5 the time-pressure histories show a consistently low pressure over the same time period, with pressure recovery and the influence of turbulent fluctuation being significantly delayed. Thereafter, the amplitude in the turbulent fluctuations increases with pressure ratio. The interplay between the pressure recovery and frequency of turbulent pressure oscillations will consequently influence the final cavity collapse conditions. Relating these macro-scale hydrodynamic behaviour to the optimum pressure ratio in terms of final reactor performance by no means straightforward however; and is not a straightforward function of the level of cavitational activity; experimental degradation studies consistently exhibit a maxima in reactor performance with respect to pressure ratio $[2,3,48,49]$. Using similar geometry to that analysed in the present work (case3), Capocelli and co-workers [9] presented empirical data on the degradation of p-nitrophenol, and observed a peak removal percentage at a pressure ratio of 4.5 , with performance decreasing at higher pressure ratios. The cavity trajectories at different pressure ratios (Figure 10) indicate a significant change in the turbulent pressure histories experienced by individual cavities between pressure ratios of $4 \& 5$, with frequencies and pressure recovery rates both decreasing. This suggests that turbulence kinetic energy and eddy frequency are potentially important parameters in determining optimum reactor performance, in addition to the rate of pressure recovery. 


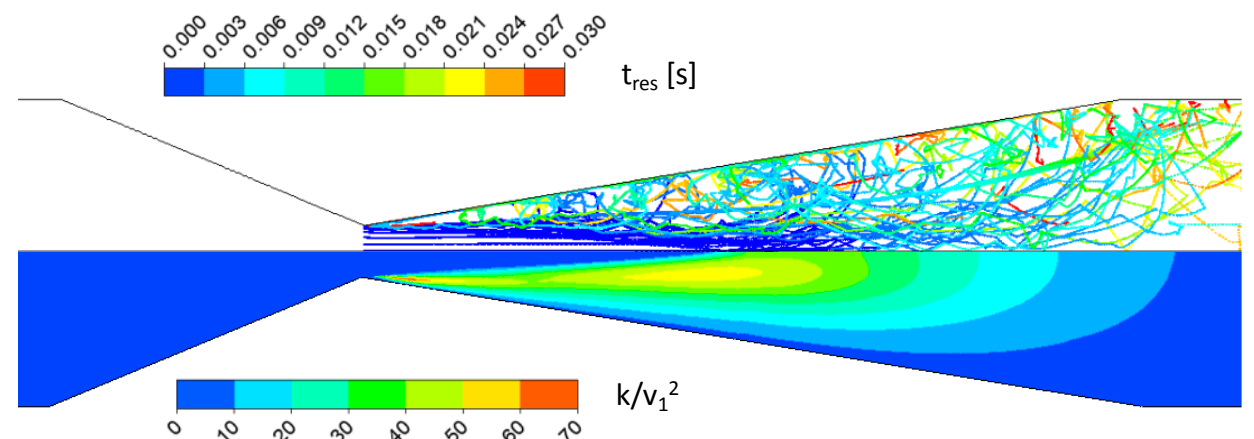

Figure 10a: Case 3, discrete cavity trajectories \& turbulent dispersion

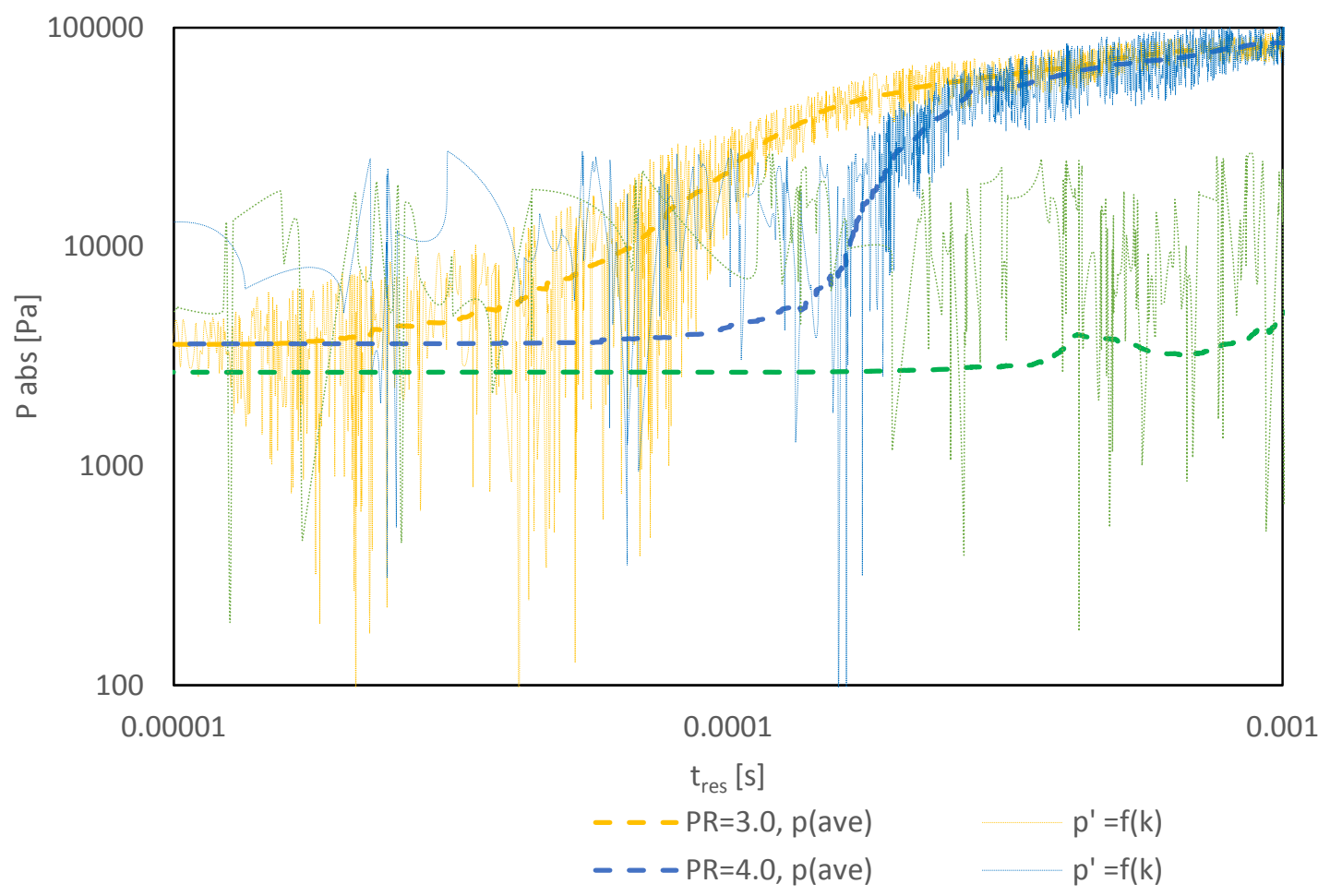

Figure 10b: Case 3, discrete cavity time - pressure histories

Figure 10: Case 3, discrete cavity predictions

\subsection{Influence of venturi l/d ratio}

The effect of throat section length has been investigated for designs having $I / d$ ratios of 1.0, $2.0 \& 3.0$, as well as the value in the baseline (Case 3 ) of 0.0. The minimum pressure in the device against pressure ratio for each configuration was examined to identify cavitation inception (see Figure 11). Based on this, predicted variation of cavitation inception with $\mathrm{l} / \mathrm{d}$ ratio is shown in Figure 12Error! Reference source not found.. Although inception numbers all lie close to one, aspect ratio has some influence on the inception point, with a maximum at an $\mathrm{l} / \mathrm{d}$ ratio of 1.0. Although the inception point is relatively consistent, increasing the $l / d$ ratio has the effect of delaying the pressure recovery, and the low-pressure region is maintained through the length of the venturi throat section (see Figure 13). Subsequently, the predicted extent of the vapour cavity is shown to increase with increasing $I / d$ ratio under matching operating pressure conditions (Figure 14 ). Generally, with $\mathrm{l} / \mathrm{d}$ ratios 
$1>0$, there are two initiation sites for cavity formation; the initial edge of the throat entry, and a second site at

2 throat exit / diffuser entry at which a second, larger cavity forms.

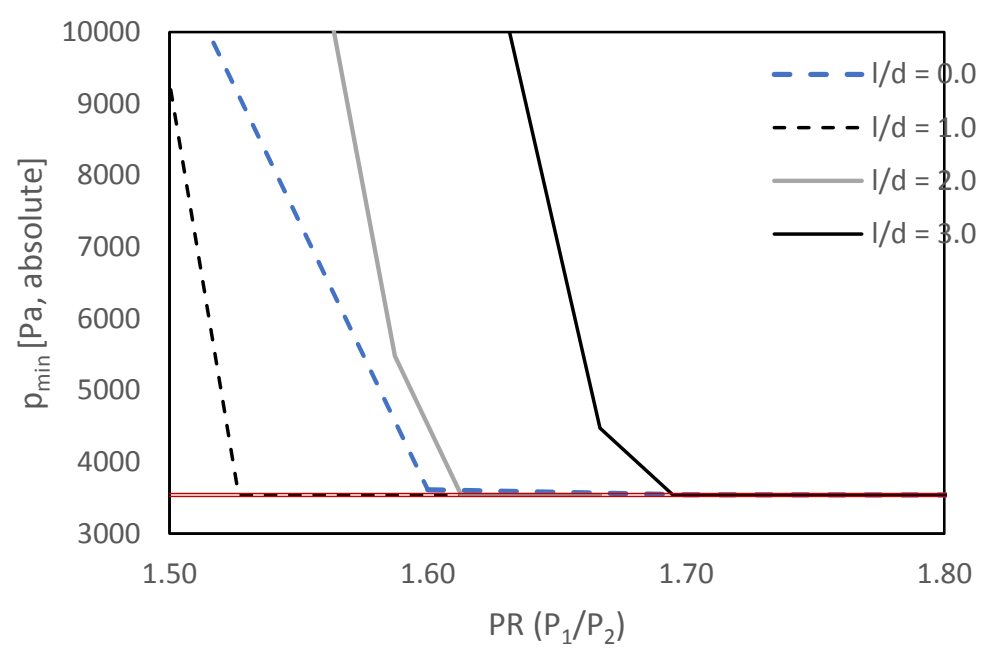

Figure 11: Minimum predicted pressure against I/d ratio

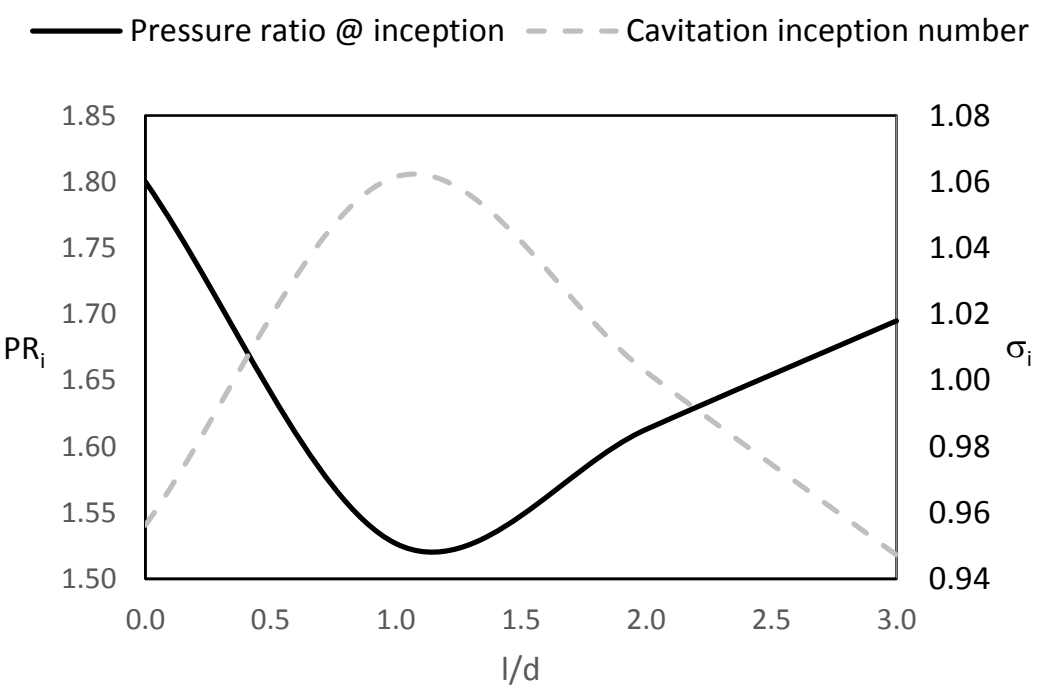

Figure 12: Cavitation inception versus $\mathrm{l} / \mathrm{d}$ ratio

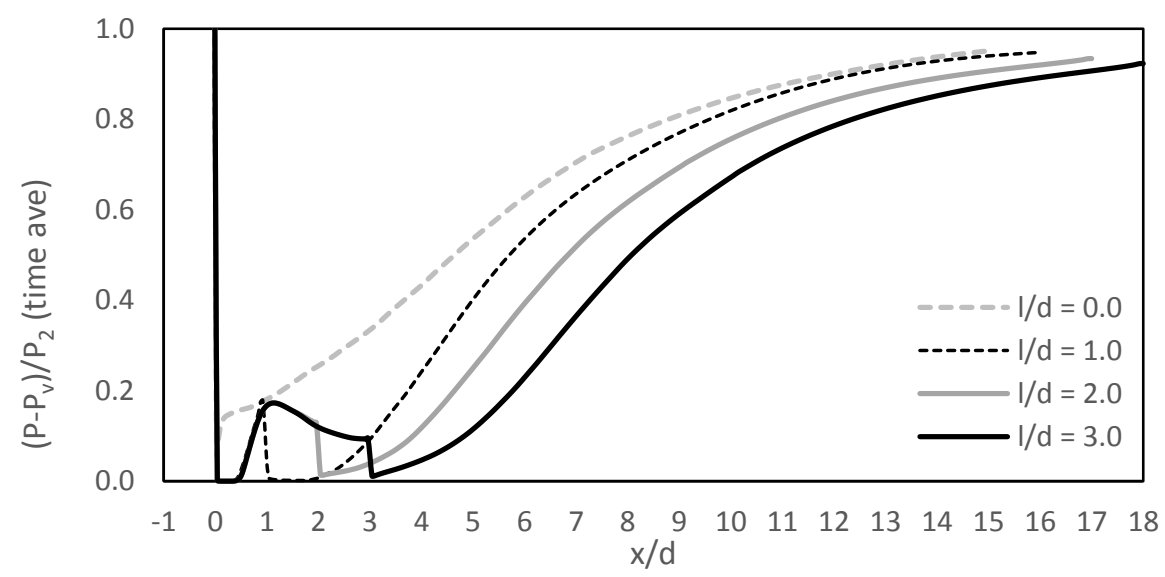

Figure 13: Static pressure vs distance along outer wall with varying $\mathrm{l} / \mathrm{d}$ ratio 

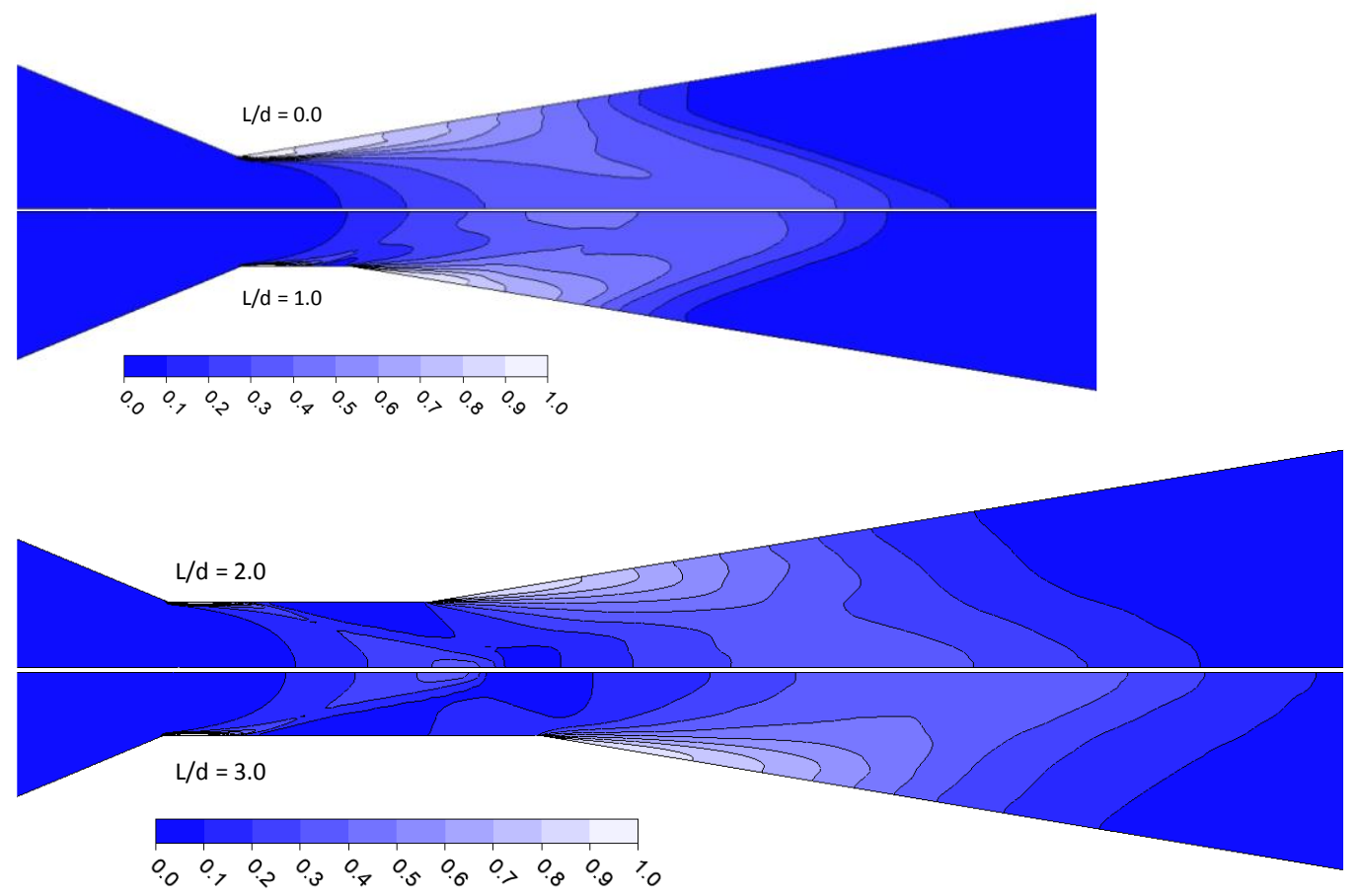

Figure 14: Variation in vapour cavity extent and shape with $\mathrm{l} / \mathrm{d}$ ratio $(P R=5.0)$

4

\subsection{Influence of diffuser angle}

6 The effect of diffuser angle has been investigated for designs having half angles, $\beta=7.25,9.2,12.5 \& 15^{\circ}$. The minimum pressure in the device against pressure ratio for each configuration was again examined to identify cavitation inception (see Figure 15). Based on this, predicted variation of cavitation inception with I/d ratio is shown in Figure 16. Wall angle is shown to significantly influence the inception point, with larger wall angles found to lead to delayed onset and growth of the predicted vapour cavity, with inception number reducing to 0.45 with a wall half angle of $15^{\circ}$. The time averaged pressure vs distance plots Figure 17 show that smaller divergence angles act to delay pressure recovery, increasing the time discrete cavities spend in the low pressure / vapour pressure region of the device. The corresponding variation in predicted vapour cavity extent and shape is presented in Figure 18.

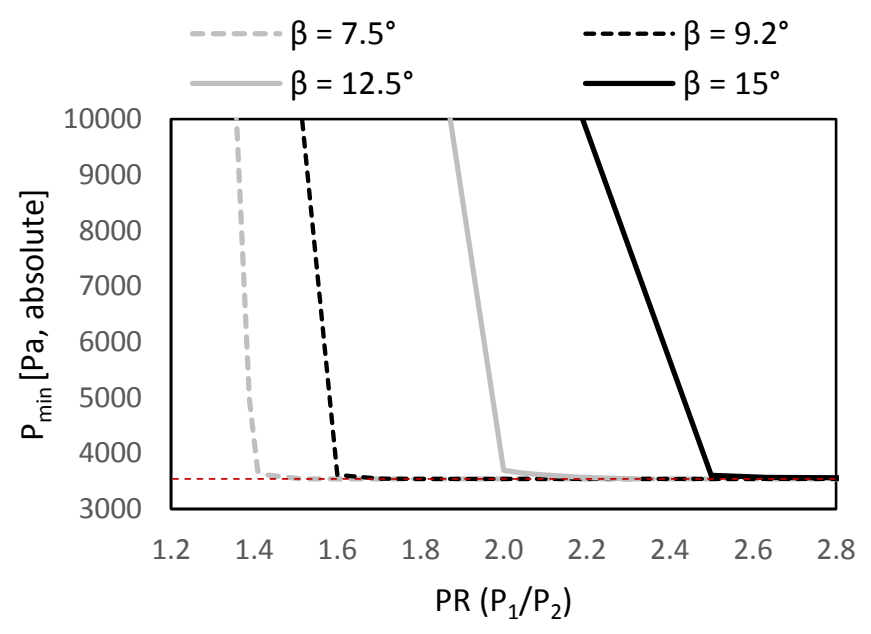


Figure 15: Minimum pressure versus diffuser angle

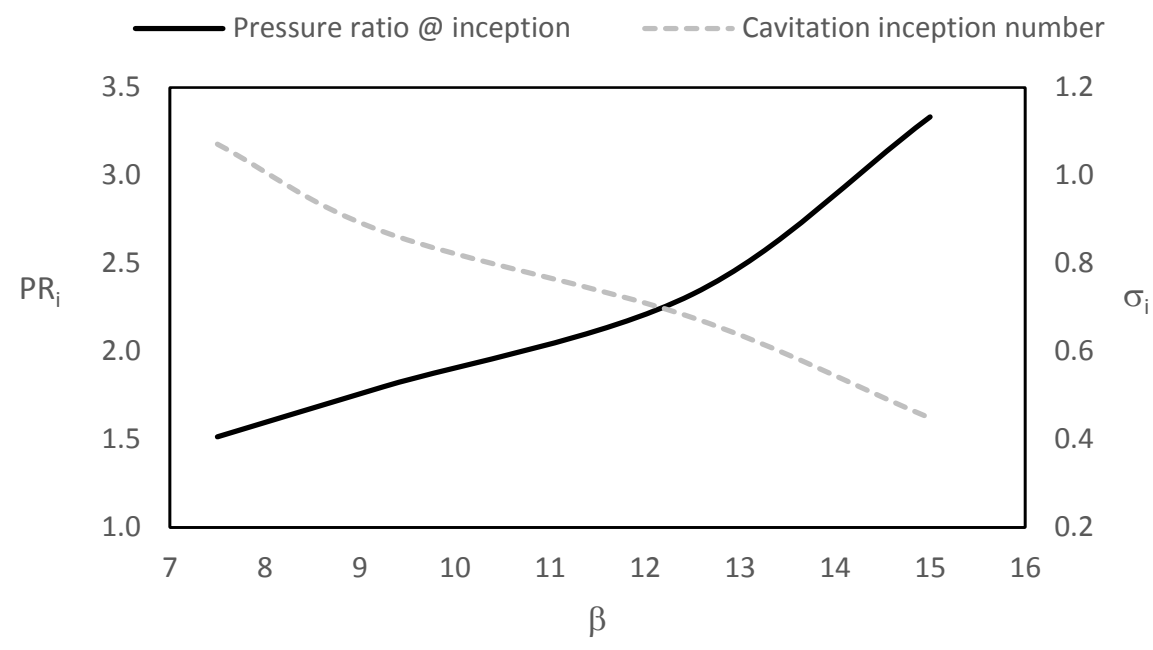

Figure 16: Cavitation inception versus diffuser angle

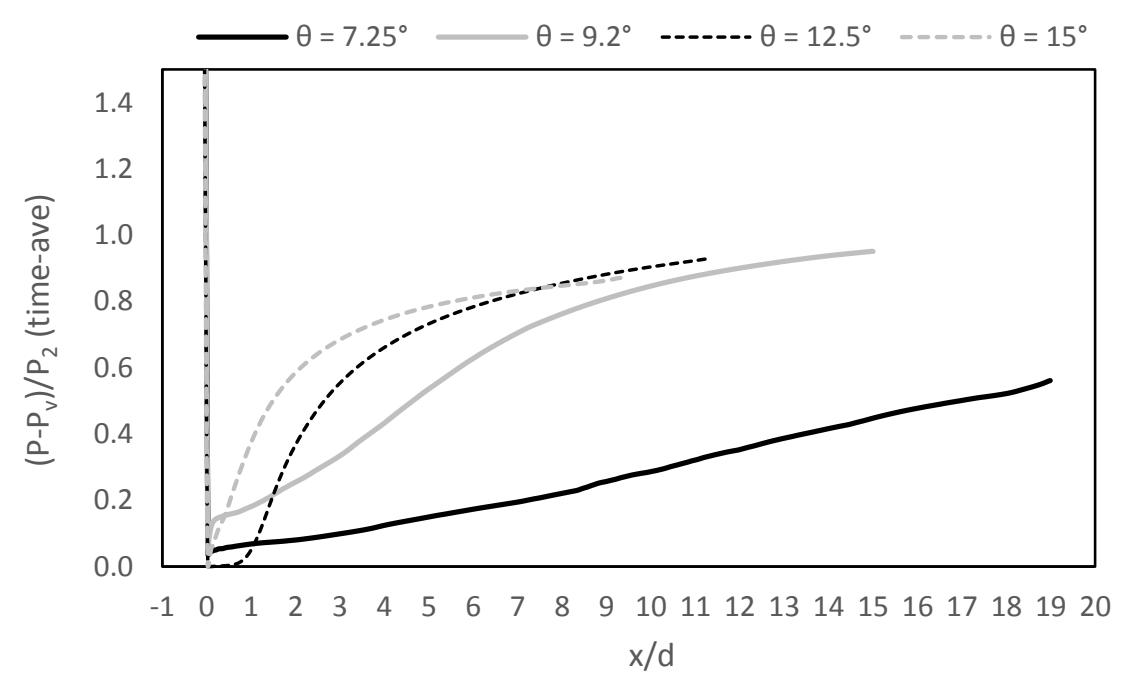

Figure 17: Static pressure vs distance along outer wall with varying diffuser angle

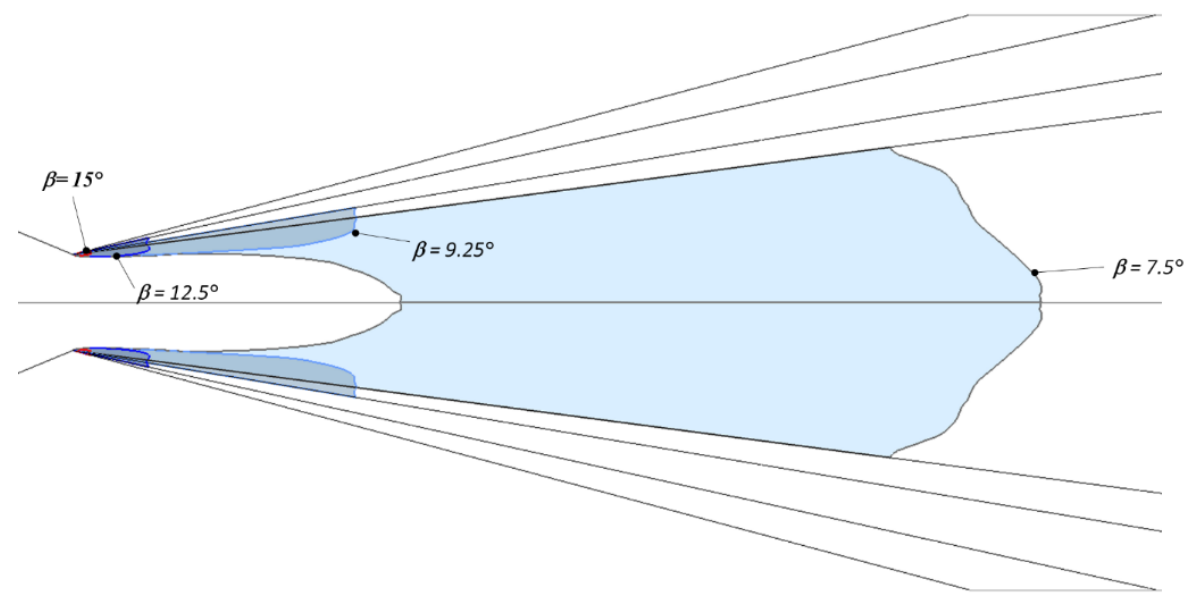

Figure 18: Predicted cavity shape and size with varying diffuser wall angles $(P R=5.0)$ 
$2 \quad 3.7$ Discrete cavity trajectories through different venturi designs

3 Figure 19 compares the discrete cavity time - pressure histories between different venturi designs, highlighting 4 the differences in the conditions created by both reducing the diffuser angle and lengthening the throat. 5 Reducing the diffuser wall angle is shown to delay pressure recovery, and also reduce the level of turbulent fluctuations initially experienced by the cavities. Similarly, increasing l/d ratio is shown to result in cavities initially experiencing a relatively low static pressure, with low frequency turbulent fluctuations over their initial lifetime. As the cavities then move further through the diffuser, they experience an increase in the frequency and amplitude of turbulent pressure fluctuations. Bubble growth is likely to be strongly dependent on these initial pressure recovery rates, and as such these results indicate the importance of these design parameters in controlling the final collapse conditions.

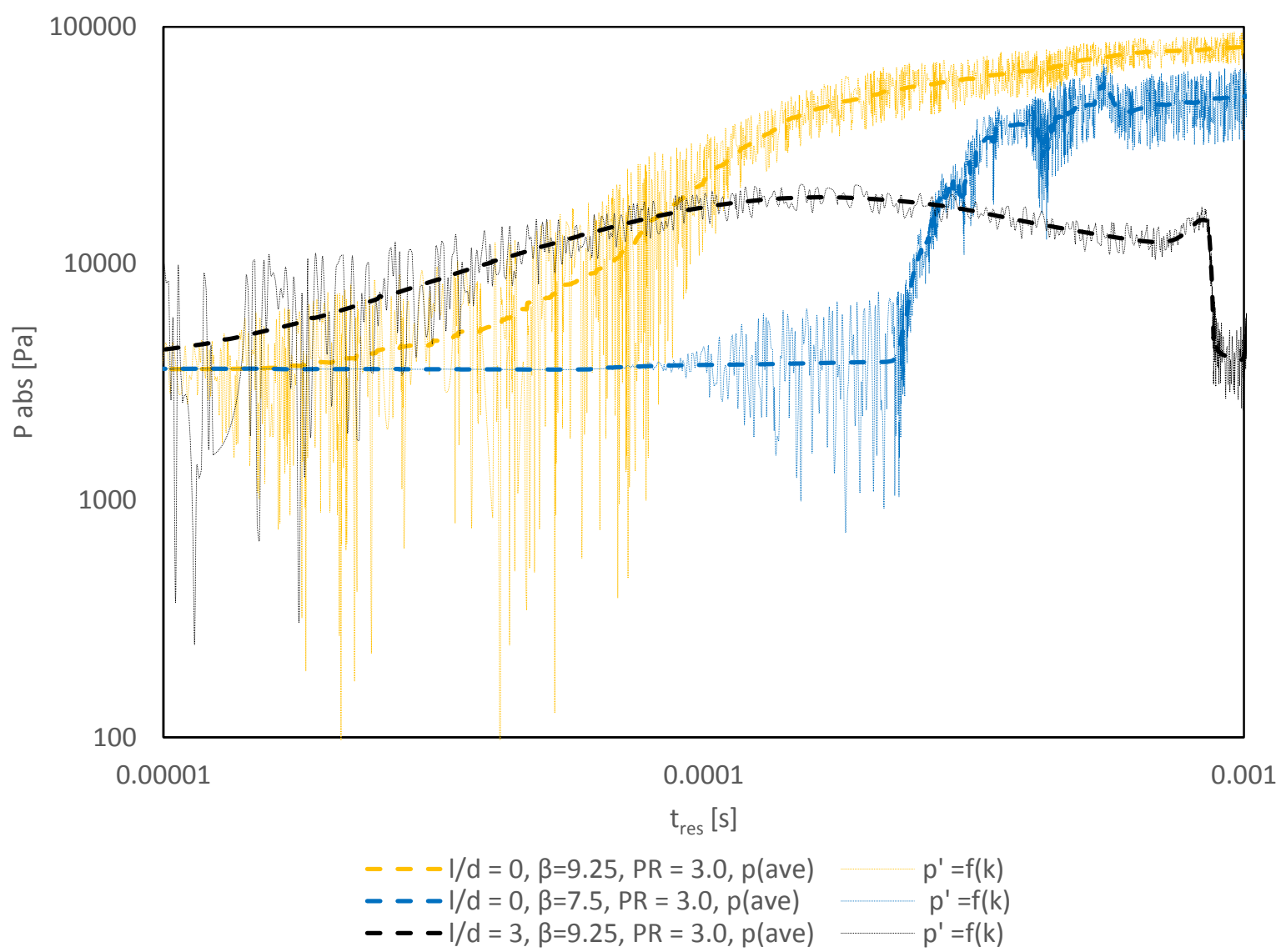

Computational fluid dynamics models were developed to simulate cavitating flow through various venturi designs. Influence of key design and operating parameters were investigated. The simulated results on flow rate versus pressure drop characteristics and cavitation inception were compared with experimental data, showing 
good agreement. The numerical model was used to decipher trends in the variation of cavitation onset and extent with varying design inputs. With respect to cavitation inception, this study suggests that an optimum configuration exists which offers a minimum power input with a low diffuser angle $\left(7.5^{\circ}\right)$ and $1 / d$ ratio of 1.0. Beyond inception point, published experimental data suggests an optimum level of cavitation activity for maximum reactor performance $[2,3,48,49]$; suggesting a corresponding optimum combination of turbulent pressure frequencies and amplitudes to maximise cavitation intensity. The results presented in the present work in terms of instantaneous pressure-time histories, determined from detailed multi-phase flow fields at device scale, are another important step towards coupling macro-hydrodynamics to final reactor performance. The presented model and results will therefore be useful to evolve optimum design parameters to achieve desired levels of cavitational activity for given flow rate / pressure ratio requirements.

The key findings of this investigation are as follows:

- The cavitation model of Singhal [41] is able to describe two phase flow in the venturi throughout the cavitating regime. Comparison with experimental data shows good agreement in predicted pressure ratio versus flow rate behaviour in the cavitating regime.

- Of the design parameters investigated in this work, the diffuser angle has the most pronounced influence on cavitation inception and extent. Smaller wall angles have the effect of encouraging cavitation to initiate at lower pressure ratios. The largest wall angle of $15^{\circ}$ considered in this work led to a reduction in cavitation inception number to 0.45 .

- Varying $\mathrm{l} / \mathrm{d}$ ratio has a less pronounced effect on cavitation inception. Increasing the $\mathrm{l} / \mathrm{d}$ ratio was found to delay pressure recovery, suggesting that this parameter may play an important role in controlling bubble growth and the final collapse conditions.

- Trajectory simulations indicate that mean pressure recovery rates are reduced with increasing pressure ratio. Furthermore, increasing the pressure ratio has been shown to significantly reduce the initial frequency of the turbulent pressure fluctuations experienced by the cavities.

The models and results presented in this work offer a means to link single cavity simulations to the output from CFD models, and thus compare the collapse pressures and temperatures obtained with different geometries and process inputs. These results will be discussed separately. The presented approach and results provide useful insights for designing and optimising venturi as hydrodynamic cavitation devices.

\section{Acknowledgements}

The authors would like to gratefully acknowledge Professor Marina Prisciandaro and the group at University of L'Aquila for providing the experimental pressure drop versus flow rate data used in this paper (Case 3 ). 


\section{Nomenclature}

$4 \quad k \quad$ Turbulent kinetic energy

$5 \quad l \quad$ Venturi throat length

$6 n$ Bubble number density

$7 \quad u, v \quad$ Velocities in the $\mathrm{x}, \mathrm{y}$ co-ordinates

$8 V_{r e l}, v_{c h}$ Relative velocity, characteristic velocity

$9 x$ Distance

$10 D$ Venturi pipe internal diameter

$11 P$ Pressure

$12 P R \quad$ Pressure ratio (inlet / outlet)

$13 T_{L} \quad$ Particle eddy lifetime

14 We Webber number

16 Greek symbols:

$17 \alpha \quad$ Volume fraction

$18 \varepsilon \quad$ Turbulence dissipation rate

$19 \beta \quad$ Venturi diffuser half-angle

$20 \sigma, \sigma_{i} \quad$ Cavitation number, cavitation number at inception

$21 \rho$ Density

$22 \omega \quad$ Turbulence dissipation rate

$23 \zeta \quad$ Normally distributed random number

$24 \Gamma \quad \Gamma \quad$ Diffusivity

25

26 Subscripts:

271 Inlet / upstream location

282 Outlet / downstream recovered pressure location

29 B Bubble

$30 t \quad$ Venturi throat

$31 \quad$ Liquid

$32 v \quad$ Vapor

$33 g \quad$ Non condensable gas (NCG)

$34 m$ Mixture

$35 p \quad$ Particle 
3 [1] Ranade V V and Bhandari V M 2014 Industrial Wastewater Treatment, Recycling and Reuse (Elsevier)

4 [2] Musmarra D, Prisciandaro M, Capocelli M, Karatza D, lovino P, Canzano S and Lancia A 2016 Degradation of ibuprofen by hydrodynamic cavitation: Reaction pathways and effect of operational parameters Ultrason. Sonochem. 29 76-83 Capocelli M, Prisciandaro M, Lancia A and Musmarra D 2015 Cavitational reactor for advanced treatment of contaminated water: the effect of recovery pressure Desalin. Water Treat. 55 3172-7

[4] Zupanc M, Kosjek T, Petkovšek M, Dular M, Kompare B, Širok B, Blažeka Ž and Heath E 2013 Removal of

[5] Gogate P R and Pandit A B 2004 A review of imperative technologies for wastewater treatment I:

[6] Dular M, Griessler-Bulc T, Gutierrez-Aguirre I, Heath E, Kosjek T, Krivograd Klemenčič A, Oder M, pharmaceuticals from wastewater by biological processes, hydrodynamic cavitation and UV treatment Ultrason. Sonochem. 20 1104-12 Petkovšek M, Rački N, Ravnikar M, Šarc A, Širok B, Zupanc M, Žitnik M and Kompare B 2016 Use of hydrodynamic cavitation in (waste)water treatment Ultrason. Sonochem. 29 577-88

[7] Kalumuck K M and Chahine G L 1998 the Use of Cavitating Jets To Oxidize Organic Compounds in Water Proc. FJIDSM ASME Fluids Eng. Div. Summer Meet. June -,, Washington, DC 122 1-10

[10] Ghayal D, Pandit A B and Rathod V K 2013 Optimization of biodiesel production in a hydrodynamic

[11] Kelkar M A, Gogate P R and Pandit A B 2008 Intensification of esterification of acids for synthesis of

[12] Maddikeri G L, Gogate P R and Pandit A B 2014 Intensified synthesis of biodiesel using hydrodynamic cavitation reactors based on the interesterification of waste cooking oil Fuel 137 285-92

[13] Terán Hilares R, de Almeida G F, Ahmed M A, Antunes F A F, da Silva S S, Han J I and Santos J C dos 2017 Hydrodynamic cavitation as an efficient pretreatment method for lignocellulosic biomassA parametric study Bioresour. Technol. 235 301-8

[14] Nakashima K, Ebi Y, Shibasaki-Kitakawa N, Soyama H and Yonemoto T 2016 Hydrodynamic Cavitation Reactor for Efficient Pretreatment of Lignocellulosic Biomass Ind. Eng. Chem. Res. 55 1866-71

[15] Madison M J, Coward-Kelly G, Liang C, Karim M N, Falls M and Holtzapple M T 2017 Mechanical pretreatment of biomass - Part I: Acoustic and hydrodynamic cavitation Biomass and Bioenergy 98 13541

6] Carpenter J, George S and Saharan V K 2017 Low pressure hydrodynamic cavitating device for producing highly stable oil in water emulsion: Effect of geometry and cavitation number Chem. Eng. Process. 
Process Intensif. 116 97-104

[17] Montusiewicz A, Pasieczna-Patkowska S, Lebiocka M, Szaja A and Szymańska-Chargot M 2017 Hydrodynamic cavitation of brewery spent grain diluted by wastewater Chem. Eng. J. 313 946-56

[18] Albanese L, Ciriminna R, Meneguzzo F and Pagliaro M 2017 Beer-brewing powered by controlled hydrodynamic cavitation: Theory and real-scale experiments J. Clean. Prod. 142 1457-70

[19] Arrojo S, Benito Y and Martínez Tarifa A 2008 A parametrical study of disinfection with hydrodynamic cavitation Ultrason. Sonochem. 15 903-8

[20] Vichare B N P, Gogate P R and Pandit A B 2000 Full Papers Optimization of Hydrodynamic Cavitation Using a Model Chem. Eng. Technol. 23 683-90

[21] Randall L N 1952 Rocket Applications of the Cavitating Venturi J. Am. Rocket Soc. 22 28-38

[22] Stutz B and Reboud J L 1997 Experiments on unsteady cavitation Exp. Fluids 22 191-8

[23] Xu C, Heister S D and Field R 2002 Modeling Cavitating Venturi Flows J. Propuls. Power 18 1227-34

[24] Ulas A 2006 Passive flow control in liquid-propellant rocket engines with cavitating venturi Flow Meas. Instrum. 17 93-7

[25] Sayyaadi H 2010 Instability of the cavitating flow in a venturi reactor Fluid Dyn. Res. 42

[26] Zhu J, Xie H, Feng K, Zhang X and Si M 2017 Unsteady cavitation characteristics of liquid nitrogen flows through venturi tube Int. J. Heat Mass Transf. 112 544-52

[27] Zhao L, Mo Z, Sun L, Xie G, Liu H, Du M and Tang J 2017 A visualized study of the motion of individual bubbles in a venturi-type bubble generator Prog. Nucl. Energy 97 74-89

[28] Sato K, Taguchi Y and Hayashi S 2013 High Speed Observation of Periodic Cavity Behavior in a Convergent-Divergent Nozzle for Cavitating Water Jet 2013 102-7

[29] Abdulaziz A M 2014 Performance and image analysis of a cavitating process in a small type venturi Exp. Therm. Fluid Sci. 53 40-8

[30] Long X, Zhang J, Wang J, Xu M, Lyu Q and Ji B 2017 Experimental investigation of the global cavitation dynamic behavior in a venturi tube with special emphasis on the cavity length variation Int. J. Multiph. Flow $89290-8$

[31] Brinkhorst S, Lavante E von and Wendt G 2017 Experimental and numerical investigation of the cavitation-induced choked flow in a herschel venturi-tube Flow Meas. Instrum. 54 56-67

[32] Patil P N, Bote S D and Gogate P R 2014 Degradation of imidacloprid using combined advanced oxidation processes based on hydrodynamic cavitation Ultrason. Sonochem. 21 1770-7

[33] Pawar S K, Mahulkar A V and Pandit A B 2017 Sonochemical Effect Induced by Hydrodynamic Cavitation : Comparison of Venturi / Orifice Flow Geometries 63 4705-16

[34] Moholkar V S, Senthil Kumar P and Pandit A B 1999 Hydrodynamic cavitation for sonochemical effects Ultrason. Sonochem. 6 53-65

[35] Capocelli M, Musmarra D, Prisciandaro M and Industriale I 2014 Chemical Effect of Hydrodynamic Cavitation : Simulation and Experimental Comparison 00

[36] Hickel S 2015 DNS and LES of two-phase flows with cavitation ERCOFTAC Ser. 20 595-604

[37] Menter F R 1994 Two-equation eddy-viscosity turbulence models for engineering applications AIAA J. 
2 [38] Ansys 2016 Ansys Inc, FLUENT 17.0 Theory Guide

3 [39] Schnerr G H and Sauer J 2001 Physical and Numerical Modeling of Unsteady Cavitation Dynamics Fourth Int. Conf. Multiph. Flow 1-12

[40] Zwart P J, Gerber A G and Belamri T 2004 A Two-Phase Flow Model for Predicting Cavitation Dynamics Fiifth International Conference on Multiphase Flow, Yokohama, Japan

7 [41] Singhal A K, Athavale M M, Li H and Jiang Y 2002 Mathematical Basis and Validation of the Full Cavitation Model J. Fluids Eng. 124617

[42] Ebrahimi B, He G, Tang Y, Franchek M, Liu D, Pickett J, Springett F and Franklin D 2017 Characterization of high-pressure cavitating flow through a thick orifice plate in a pipe of constant cross section Int. J. Therm. Sci. 114 229-40

[43] Patankar S V 1980 Numerical Heat Transfer and Fluid Flow (Hemisphere, Washington D.C.)

Bardina, J.E., Huang, P.G., Coakley T J 1997 Turbulence modelling validation AIAA-1997-2121

Reboud J-L, Stutz B and Coutier O 1998 Two-phase flow structure of cavitation: Experiment and

[46] Coutier-Delgosha O, Fortes-Patella R and Reboud J L 2003 Evaluation of the Turbulence Model Influence

[47] Charriere B, Decaix J and Goncalves E 2015 A comparative study of cavitation models in a Venturi flow

[48] Gore M M, Saharan V K, Pinjari D V., Chavan P V. and Pandit A B 2014 Degradation of reactive orange 4 dye using hydrodynamic cavitation based hybrid techniques Ultrason. Sonochem. 21 1075-82

Patil P N and Gogate P R 2012 Degradation of methyl parathion using hydrodynamic cavitation: Effect of operating parameters and intensification using additives Sep. Purif. Technol. 95 172-9 